\title{
Evidence for AGN Feedback in Galaxy Clusters and Groups
}

\author{
Myriam Gitti, ${ }^{1,2,3}$ Fabrizio Brighenti, ${ }^{4}$ and Brian R. McNamara ${ }^{2,5,6}$ \\ ${ }^{1}$ INAF-Osservatorio Astronomico di Bologna, Via Ranzani 1, 40127 Bologna, Italy \\ ${ }^{2}$ Harvard-Smithsonian Center for Astrophysics, 60 Garden Street, Cambridge MA 02138, USA \\ ${ }^{3}$ INAF-Istituto di Radioastronomia di Bologna, Via Gobetti 101, 40129 Bologna, Italy \\ ${ }^{4}$ Dipartimento di Astronomia, Università di Bologna, Via Ranzani 1, 40127 Bologna, Italy \\ ${ }^{5}$ Physics and Astronomy Department, University of Waterloo, 200 University Avenue West, Waterloo, ON, Canada N2L 2G1 \\ ${ }^{6}$ Perimeter Institute for Theoretical Physics, 31 Caroline Street North, Waterloo, ON, Canada N2L 2Y5
}

Correspondence should be addressed to Myriam Gitti, myriam.gitti@oabo.inaf.it

Received 29 July 2011; Accepted 14 September 2011

Academic Editor: Angela Bongiorno

Copyright ( $) 2012$ Myriam Gitti et al. This is an open access article distributed under the Creative Commons Attribution License, which permits unrestricted use, distribution, and reproduction in any medium, provided the original work is properly cited.

The current generation of flagship X-ray missions, Chandra and XMM-Newton, has changed our understanding of the so-called "cool-core" galaxy clusters and groups. Instead of the initial idea that the thermal gas is cooling and flowing toward the center, the new picture envisages a complex dynamical evolution of the intracluster medium (ICM) regulated by the radiative cooling and the nongravitational heating from the active galactic nucleus (AGN). Understanding the physics of the hot gas and its interplay with the relativistic plasma ejected by the AGN is key for understanding the growth and evolution of galaxies and their central black holes, the history of star formation, and the formation of large-scale structures. It has thus become clear that the feedback from the central black hole must be taken into account in any model of galaxy evolution. In this paper, we draw a qualitative picture of the current knowledge of the effects of the AGN feedback on the ICM by summarizing the recent results in this field.

\section{Introduction}

The physics of the intracluster medium (ICM) of clusters and groups of galaxies is complex. The current generation of X-ray satellites, Chandra and XMM-Newton, has shown indeed that it is regulated by yet poorly understood nongravitational processes beyond simple gravity, gas dynamics, and radiative cooling usually considered in the standard cold dark matter cosmological scenario [1]. In particular, an important discovery from high-resolution X-ray observations was that the amount of thermal gas radiatively cooling to low temperatures is much less than what is predicted by the standard "cooling flow" model (see [2-5] and references therein), thus radically changing our understanding of the so-called "cool-core" systems. The implication is that the central gas must experience some kind of heating due to a feedback mechanism that prevents cool cores from establishing cooling flows at the rates predicted by earlier, lowresolution X-ray observations. Establishing the source of this heating, and understanding when and how it takes place, has become a major topic of study in extragalactic astrophysics.
Based on observational evidence and theoretical modelling, the primary source of feedback has been identified in the outbursts and accompanying energy injection, likely intermittent, from the active galactic nucleus (AGN) of the dominant $\mathrm{cD}$ galaxies (e.g., [6] and references therein), which host the most massive black holes in the local Universe. AGNs manifest as central radio sources, which are commonly observed in cool-core clusters [7]. Most of the cool-core systems have highly disturbed X-ray morphologies, and radio observations clearly show that AGN jets are the cause of many of the structures revealed by the X-ray telescopes. Such surface brightness features, including apparent depressions or "cavities" in the X-ray images and sharp density discontinuities interpreted as shocks, indicate a strong interaction between the central AGN and the intracluster medium (ICM). The incidence and variety of cavities, shocks, and ripples observed both in the radio and in X-rays in the hot ICM provides direct evidence of the widespread presence of AGN-driven phenomena (e.g., [8, 9] for sample studies of clusters and groups, resp.). 
Such AGN feedback has a wide range of impacts, from the formation of galaxies, through to the explanation of the observed relation between the black hole mass and the bulge velocity dispersion (which indicates a causal connection or feedback mechanism between the formation of bulges and their central black holes, e.g., [10]), to the regulation of cool cores which explains why cooling and star formation still proceed at a reduced rate. On the other hand, the details of how the feedback loop operates are still unknown. Feedback is also required to suppress the overproduction of massive galaxies predicted by dark-matter-only simulations and to break the self-similarity of clusters (e.g., [11-13]). The nature of this feedback is therefore vital to our understanding of galaxy evolution ([14] and references therein).

After a brief discussion of the importance of galaxy clusters and their scaling relations for the study of cosmic evolution (Section 2), we overview the role of AGN feedback in structure formation (Section 3) and the basic properties of clusters in X-rays emphasizing the hot intracluster medium (Section 4). We then focus on the observational evidence of AGN feedback in action in galaxy clusters and groups (Section 5) and finally give our conclusions (Section 6). The present paper does not intend to be a comprehensive review, but aims instead at drawing a qualitative picture of the impact of the AGN feedback on the ICM addressed particularly to the novices in this field. General reviews of clusters from an Xray perspective were given by Sarazin [15] and more recently by Mushotzky [16] and Arnaud [17]. A review of clusters as cosmological probes was given by Voit [18], and cold fronts and shocks associated with cluster mergers were reviewed by Markevitch and Vikhlinin [19]. An exhaustive review of the issues of AGN heating in the hot atmospheres was recently given by McNamara and Nulsen [6].

Throughout the paper we assume a cosmology with $H_{0}=$ $70 \mathrm{~km} \mathrm{~s}^{-1} \mathrm{Mpc}^{-1}$ and $\Omega_{M}=1-\Omega_{\Lambda}=0.3$, where not specified otherwise.

\section{Clusters of Galaxies as Cosmological Probe}

The existence of clusters of galaxies and of other cosmic structures demonstrates that the Universe is not perfectly homogeneous. The matter density of the primordial Universe must have been slightly inhomogeneous, with overdense perturbations which deviate from the mean density. In the so-called "Concordance Model" largely accepted today as the standard cold dark matter cosmological scenario, cosmic structures like galaxies and clusters of galaxies originate from the gravitational instability of these primordial density fluctuations. The formation of structures from perturbations in the density distribution of cold dark matter is a hierarchical process. Small subclumps of matter are the first to deviate from the Hubble flow, to collapse and to experience gravitational relaxation because the density perturbations have larger amplitudes on smaller mass scales. These small objects then undergo a merging process to form larger and larger structures, up to the clusters of galaxies [1].

Galaxy clusters trace the high-density tail of the primordial field of dark matter density perturbations, and their numerical density as a function of redshift, $z$, is highly sensitive to the specific cosmological scenario (e.g., [20] and references therein). Therefore, if one builds the so-called "cluster mass function" $n_{M}(M)$, that is, the number density of clusters with mass greater than $M$ in a comoving volume element and determines its evolution with redshift, it is possible to constrain the main cosmological parameters from the comparison between the observations and the theory predictions. A complete understanding of the details of the process of hierarchical merging would require accurate numerical simulations. However, many fundamental aspects can be illustrated by spherically symmetric, simplified models of cluster formation. In particular, the combination of spherical top-hat collapse models with the growth function for linear perturbations (assumed to be gaussian) has led to a variety of semianalytical methods to express the cluster mass function in terms of cosmological parameters (the seminal work in this field is by [21]).

Therefore, the comparison between the theoretical mass function and the mass function determined from observations allows one to constrain the main cosmological parameters, although with a degeneracy between the matter density parameter, $\Omega_{M}$, and the power spectrum normalization of the perturbations within a comoving sphere of radius $8 \mathrm{~h}^{-1} \mathrm{Mpc}, \sigma_{8}$. Such a degeneracy can be broken by studying the redshift evolution of the mass function, which is highly sensitive to $\Omega_{M}$, by taking into account the evolution of the observables (see the reviews by $[18,20,22]$ and references therein). The accuracy of the cosmological parameter measurements is currently limited by uncertainties in the relations between cluster masses and the observable properties that trace these masses, such as luminosity or temperature. In order to measure the mass function from a large sample of clusters is indeed necessary to link the mass to these quantities which are easily observable.

In this context are very useful the so-called "self-similar scaling relations," derived naturally by considering that the cosmological structures originate from scale-free density perturbations and that the thermodynamical properties of the ICM are determined by scale-free gravity only [23]. Under these assumptions, galaxy clusters of different masses may be considered as a scaled version of each other. The density of each dark matter halo, $\rho_{\mathrm{DM}}$, is proportional to the critical density of the Universe at the cluster's redshift, $\rho_{c, z}$, through the so-called "overdensity" $\Delta=\rho_{\mathrm{DM}} / \rho_{c, z}$, where $\rho_{c, z}=3 H_{z}^{2} / 8 \pi G$, and the expression for the Hubble constant at redshift $z$ in a flat $\Lambda$ CDM Universe is (e.g., [24]): $H_{z}=H_{0} \sqrt{\Omega_{m}(1+z)^{3}+\Omega_{\Lambda}} \equiv H_{0} E(z)$. Therefore, all clusters should have the same properties when rescaled by $\Delta$.

If we define the mass $M$ as the mass $M_{\Delta}$ inside the radius $R_{\Delta}$ at a given overdensity $\Delta$, we obtain: $M_{\Delta} \propto \rho_{c, z} \cdot \Delta \cdot R_{\Delta}^{3} \propto$ $\rho_{c, 0} \cdot E(z)^{2} \cdot \Delta \cdot R_{\Delta}^{3}$, and thus we get to the $M-R$ relation in the form

$$
R \propto M^{1 / 3} \cdot E(z)^{-2 / 3}
$$

During cluster formation, the gravitational collapse of the diffuse gas in the potential well of the dark matter halo heats the gas itself at the virial temperature of the potential well that confines it: $T_{\text {vir }} \sim G M \mu m_{p} / k R \sim 10^{8} \mathrm{~K}$, where $M$ 
is the total mass, $k$ is the Boltzmann constant, $\mu \sim 0.6$ is the mean molecular weight and $R$ is the virial radius. The gas is thus heated to X-ray emitting temperatures and becomes a plasma in hydrostatic equilibrium whose emissivity is proportional to the square of its density (see Section 4). The virial temperature of an isothermal sphere of mass $M$ is $k T \propto M / R \propto M^{2 / 3} \cdot E(z)^{2 / 3}$, leading to the $M-T$ relation in the form:

$$
M \propto T^{3 / 2} \cdot E(z)^{-1} .
$$

From these relations it is possible to derive the relation between temperature and luminosity emitted by the hot gas through bremsstrahlung emission: $L_{X} \propto \rho^{2} \cdot \Lambda \cdot V$, where $\rho$ is the average gas density and $\Lambda$ is the cooling function, that in the bremsstrahlung regime is $\propto T^{1 / 2}$ (see Section 4). Assuming that the gas distribution traces the dark matter distribution, $\rho \propto \rho_{\mathrm{DM}} \propto \rho_{c, z}$, we obtain: $L_{X} \propto \rho \cdot T^{1 / 2} \cdot M \propto$ $\rho_{o} \cdot E(z)^{2} \cdot T^{1 / 2} \cdot M \propto E(z)^{2} \cdot T^{1 / 2} \cdot T^{3 / 2} \cdot E(z)^{-1}$, thus deriving the $L-T$ relation in the form:

$$
L_{X} \propto T^{2} \cdot E(z) .
$$

By combining the $M-T$ relation (2) with the $L-T$ relation (3), we can finally derive the $M-L$ relation that links the mass directly to the observable luminosity: $L_{X} \propto$ $\left[M^{2 / 3} E(z)^{2 / 3}\right]^{2} E(z)$, finding

$$
M \propto L_{X}^{3 / 4} \cdot E(z)^{-7 / 4} .
$$

In principle, once calibrated with simulations and/or observations, these scaling relations provide a method to link the mass of clusters to observables under the assumption that the process of structure formation is guided by gravity alone. On the other hand, deviations from these relations testify the presence of physical processes more complex than gravitational dynamics only, which modify the thermodynamical properties of the diffuse baryons and therefore the relations between observables and cluster masses. In particular, a number of observational measurements seems to indicate that the $L-T$ relation is steeper than that predicted by selfsimilar models and is in the form $L \propto T^{2.5-3}$ (e.g., [2530], see also Section 5.6). This observed breaking of the scaling relation has been ascribed to the presence of some excess entropy in the gas due to primordial nongravitational heating before the cluster virialization $[31,32]$ and is one of the strongest evidence for nongravitational processes acting in the ICM.

The main source of uncertainty in the determination of cosmological parameters from studies of cluster samples arises then from the uncertainty in the normalization, shape, and evolution of the relationships that relate the cluster masses to the observables. In order to understand better such relations, it is essential to investigate how the structure formation and AGN feedback affect the evolution of what we can observe, that is, the baryons in clusters.

\section{Role of AGN Feedback in Galaxy Evolution}

One of the main problems of the current cosmological model is why so few baryons have formed stars $[33,34]$. Numerical simulations of cosmological structure formation that include the hydrodynamics of baryons and the radiative cooling processes predict that $\gtrsim 20 \%$ of the baryons should have condensed into galaxies, but only $\lesssim 10 \%$ have been observed in the form of stars (e.g., [35]). In particular, simulations that include only gravitational heating predict an excessive cooling of baryons that results in a population of galaxies which are too massive and too bright with respect to the ones observed, thus failing to reproduce the truncation of the high-luminosity end of the galaxy luminosity function $[11,36]$.

Instead of residing in the $\mathrm{cD}$ galaxies as predicted by simulations, most baryons are observed in the hot ICM. This problem may find a solution in the nongravitational heating supplied by supernovae (SN) and active galactic nuclei (AGN). Supernovae are essential in the process of enrichment of the ICM to the metallicity level observed $[37,38]$, and from the heavy-element abundances in clusters it is estimated that during a cluster history they supply a total amount of energy of the order of $0.3-1 \mathrm{keV} /$ particle (e.g., $[39,40])$. This is not enough energy to quench cooling in massive galaxies [38], as the energy input required to explain the mass-observable relations is $\sim 1-2 \mathrm{keV} /$ particle [41-43]. Energetically, AGN heating appears to be the most likely mechanism to severely reduce the supply of gas from the hot ICM in massive galaxies and to explain the observed entropy profiles [11, 18, 44-46]. AGNs are powered by accretion of material onto a black hole $(\mathrm{BH})$, which is located at the center of each stellar spheroid (both bulges within spirals and ellipticals). Matter falling onto a black hole releases an energy of the order of $E_{\mathrm{BH}}=\epsilon M c^{2}$, where $\epsilon \approx 0.1$ is the efficiency. For supermassive black holes (SMBHs) of masses $\sim 10^{9} M_{\odot}$, the amount of energy released during their formation and growth is of the order of $E_{\mathrm{BH}} \sim 2 \times 10^{62} \mathrm{erg} \mathrm{s}^{-1}$. Even a tiny fraction $(\lesssim 1 \%)$ of the energy released within the bulge could heat and blow away its entire gas content in small systems and prevent cooling, thus explaining the lack of star formation in bulges. An extraordinary discovery obtained recently in astrophysics is the correlation between the mass of the central black hole $\left(M_{\mathrm{BH}}\right)$ and the velocity dispersion $(\sigma)$ of the galaxy's bulge used to estimate the mass of the bulge itself [10]. This "Magorrian relation" $M_{\mathrm{BH}^{-}} \sigma$ suggests that the large-scale properties of the galaxy and the smallscale properties of the black hole are related. In particular, each massive galaxy seems to host a central black hole, whose mass is $\sim 0.1-0.2 \%$ of the bulge stellar mass [47-49]. Such a correlation may arise from the fact that the central black hole is able to regulate the amount of gas available for star formation in the galaxy. The formation of black holes and the formation of bulges are closely linked. Therefore supermassive black holes can have a profound influence on the formation and evolution of galaxies. The physical process regulating these phenomena has been called "feedback," and the understanding of how it acts in detail is one of the main open issues in extragalactic astrophysics.

Clusters of galaxies are the only locations in the Universe where we can find an almost complete census of the intergalactic baryons and a very good description of their thermodynamical status and of their enrichment in heavy elements. 
Therefore, X-ray observations of the ICM can provide us with new important insights into the processes of cooling and feedback which regulate galaxy formation.

\section{Clusters of Galaxies in X-Rays and Thermal ICM}

Clusters of galaxies are the largest virialized structures in the Universe, with typical sizes of $\sim 2-4 \mathrm{Mpc}$ and total gravitational masses of $\sim 10^{14}-10^{15} M_{\odot}$. They are luminous $\mathrm{X}$-ray sources, with typical luminosities ranging from a few $\times 10^{43}$ $10^{46} \mathrm{erg} \mathrm{s}^{-1}$. As first suggested by Felten et al. [50], the X-rays from clusters are primarily thermal bremsstrahlung emission from the diffuse ICM which fills the deep potential wells and is heated to temperatures of $\sim 10^{8} \mathrm{~K}$ (where $k T=1 \mathrm{keV}$ for $T=1.16 \times 10^{7} \mathrm{~K}$ ) during the process of cluster formation.

4.1. Physical Properties of Hot Diffuse Plasma. The simple assumptions which are generally made in the study of the ionization state and X-ray line and continuum emission from a low-density hot plasma are briefly reviewed below [15].

(1) The time scale for elastic Coulomb collisions between particles in the plasma is much shorter than the age or cooling time of the plasma, therefore the free particles are assumed to have a Maxwell-Boltzmann distribution at the temperature $T$. This follows from considerations on the mean free paths of particles in a plasma without a magnetic field. The mean free path $\lambda_{\mathrm{e}}$ for an electron to suffer an energy exchange with another electron via Coulomb collisions is given by [51]:

$$
\lambda_{e}=\frac{3^{3 / 2}\left(k T_{e}\right)^{2}}{4 \pi^{1 / 2} n_{e} e^{4} \ln \Lambda},
$$

where $T_{e}$ is the electron temperature, $n_{e}$ is the electron number density, and $\Lambda$ is the ratio of largest to smallest impact parameters for the collisions $(\ln \Lambda \approx$ 38). Equation (5) assumes that the electrons have a Maxwellian velocity distribution at the electron temperature $T_{e}$. However, it can be demonstrated that if a homogeneous plasma is created in a state in which the particle distribution is non-Maxwellian, elastic collisions will cause it to relax to a Maxwellian distribution on a time scale determined by the mean free paths $[51,52]$. Electrons will achieve this equilibrium on a time scale given roughly by $t_{\mathrm{eq}}(e, e) \equiv \lambda_{e} /\left\langle v_{e}\right\rangle_{\mathrm{rms}}$, where $\left\langle v_{e}\right\rangle_{\mathrm{rms}}$ is the rms electron velocity $=\left(3 k T_{e} / m_{e}\right)^{1 / 2}$ :

$t_{\mathrm{eq}}(e, e) \approx 3.3 \times 10^{5} \mathrm{yr}\left(\frac{T_{e}}{10^{8} \mathrm{~K}}\right)^{3 / 2}\left(\frac{n_{e}}{10^{-3} \mathrm{~cm}^{-3}}\right)^{-1}$.

The time scale for Coulomb collisions between protons to bring them into kinetic equilibrium is about $t_{\mathrm{eq}}(p, p) \approx\left(m_{p} / m_{e}\right)^{1 / 2} t_{\mathrm{eq}}(e, e)$, roughly 43 times longer than that for electrons. After this time, the electrons and ions (generally assumed to be protons) would each have Maxwellian distribution, but generally at different temperatures, respectively, $T_{e}$ and $T_{i}$. The time scale for the electrons and ions to reach equipartition $T_{e}=T_{i}$ is $t_{\mathrm{eq}}(p, e) \approx$ $\left(m_{p} / m_{e}\right) t_{e q}(e, e)$, and for typical values of the ICM temperature and density is $t_{\mathrm{eq}}(p, e) \lesssim 6 \times 10^{8} \mathrm{yr}$. Since this is shorter than the age of the clusters or their large-scale cooling time (although it is comparable to or longer than the cooling time in the cores of clusters, see (21) below), the intracluster plasma can generally be characterized by a single kinetic temperature $T=T_{e}=T_{i}$, which determines the rates of all excitation and ionization processes. It is important to note that the mean free paths, that is,

$$
\lambda_{e}=\lambda_{i} \approx 23 \mathrm{kpc}\left(\frac{T_{e}}{10^{8} \mathrm{~K}}\right)^{2}\left(\frac{n_{e}}{10^{-3} \mathrm{~cm}^{-3}}\right)^{-1},
$$

are generally much shorter than the length scales of interest in clusters $(\approx 1 \mathrm{Mpc})$, and therefore the ICM can be treated as a collisional fluid, satisfying the hydrodynamic equations.

(2) At these low densities, collisional excitation and deexcitation processes are much slower than radiative decays, therefore any ionization or excitation process is assumed to be initiated from the ground state of an ion. Three (or more) body collisional processes are ignored because of the low density.

(3) Stimulated radiative transitions are not important, since the radiation field in the ICM is sufficiently dilute.

(4) At these low densities, the gas is optically thin and the transport of the radiation field can therefore be ignored.

Under these conditions, ionization and emission result primarily from collisions of ions with electrons, and collisions with other ions can be ignored. The time scales for ionization and recombination are generally considerably shorter than the age of the cluster or any relevant hydrodynamic time scale, therefore the plasma is assumed to be in ionization equilibrium (e.g., [53]). The equilibrium ionization state of a diffuse plasma depends only on the electron temperature: since in nearly all astrophysical plasmas most of the electrons originate in hydrogen and helium atoms, and these are fully ionized under the conditions considered here, the ICM is generally treated as a fully ionized plasma.

By indicating with $X, Y, Z$ the mass fraction of hydrogen, helium, and heavier elements, respectively, the corresponding atom number densities can be written in the form: $n_{\mathrm{H}}=n_{p} \equiv \rho X / m_{p}, n_{\mathrm{He}}=\rho Y / 4 m_{p}=n_{p} Y / 4 X, n_{z}=$ $\rho Z / A m_{p}=n_{p} Z / A X$, where $\rho$ is the gas density, $m_{p}$ the proton mass and $A$ the mean atomic mass number (i.e., the number of nucleons) of heavier elements. Assuming that the gas pressure $p=n k T$ is contributed only by electrons and protons, thus neglecting nuclei $\left(n=n_{e}+n_{p}\right)$, it is possible to derive the electron density $n_{e}$ in terms of the proton density 
$n_{p}$. From the expression of the number of particles contributing to the pressure, $n=2 n_{\mathrm{H}}+2 n_{\mathrm{He}}+(1 / 2) A n_{z}$, one obtains

$$
n=\left(2+\frac{1}{2} \frac{Y}{X}+\frac{1}{2} \frac{Z}{X}\right) n_{p}
$$

which for solar abundances $(X=0.71, Y=0.265$, $Z=0.025$ ) leads to $n_{e} \sim 1.2 n_{p}$. It is also possible to calculate the mean molecular weight in amu, $\mu$, such that the total number density of particles (electrons, protons and ions) is $\bar{n}=\rho / \mu m_{p}$. From the expression $\bar{n}=2 n_{\mathrm{H}}+3 n_{\mathrm{He}}+((1 / 2) A+$ 1) $n_{z}$, in the approximation $A \gg 1$, one obtains

$$
\mu=\left(2 X+\frac{3}{4} Y+\frac{1}{2} Z\right)^{-1},
$$

which for solar abundances leads to $\mu \sim 0.6$.

4.2. X-Ray Emission from the ICM. The X-ray continuum emission from a hot diffuse plasma, such as the ICM, is due primarily to two processes: thermal bremsstrahlung (freefree emission) and recombination (free-bound) emission. Processes that contribute to X-ray line emission (boundbound radiation) from a diffuse plasma include collisional excitation of valence or inner shell electrons, radiative and dielectric recombination, inner shell collisional ionization, and radiative cascades following any of these processes.

At the high temperatures, typical of clusters (in particular at $k T \gtrsim 2.5 \mathrm{keV}$ ), thermal bremsstrahlung is the predominant X-ray emission process. The bremsstrahlung emissivity at a frequency $\nu$ (defined as the emitted energy per unit time, frequency, and volume) of a plasma with temperature $T$, electron density $n_{e}$ and ion density $n_{i}$ is given by (e.g., [54]):

$$
J_{\mathrm{br}}(\nu, T)=6.8 \times 10^{-38} Z^{2} n_{e} n_{\mathrm{i}} T^{-1 / 2} e^{-h \nu / k T} \bar{g}(\nu, T),
$$

where the Gaunt factor $\bar{g}(T)$, which corrects for quantum mechanical effects and for the effect of distant collisions, is a slowly varying function of the parameters $[55,56]$. If the ICM is mainly at a single temperature, then (10) indicates that the X-ray spectrum should be close to an exponential of the frequency, as is generally observed.

The total power per unit volume emitted by thermal bremsstrahlung is obtained by integrating (10) over frequency, obtaining:

$$
J_{\mathrm{br}}(T)=1.4 \times 10^{-27} n_{e} n_{i} T^{1 / 2} Z^{2} \bar{g}(T),
$$

where $\bar{g}(T)$ is a frequency average of $\bar{g}(\nu, T)$ and is in the range 1.1 to 1.5 (choosing a value of 1.2 will give an accuracy in the estimate of $J_{\mathrm{br}}(T)$ to within about $20 \%$, [54]). For solar abundances, the emission is primarily from hydrogen and helium.

Compilations of the different emissivities for X-ray lines and continua can be found in the literature (e.g., $[57,58])$. Early detailed calculations of the X-ray spectra predicted by different models of the ICM have been given by Sarazin and Bahcall [59] and Bahcall and Sarazin [60, 61]. In these models most of the X-ray emission is thermal bremsstrahlung continuum, and the strongest lines (highest equivalent width) are in the $7 \mathrm{keV}$ iron line complex.
The frequency-integrated total emissivity at a temperature $T$ can be written as:

$$
J_{X}(T)=\Lambda(T) n_{e} n_{p} \operatorname{erg~s}^{-1} \mathrm{~cm}^{-3},
$$

where $\Lambda(T)$ is the cooling function, which depends upon the mechanism of the emission and can be represented as:

$$
\Lambda(T)=l T^{\alpha},
$$

where $-0.6 \lesssim \alpha \lesssim 0.55$; for thermal bremsstrahlung it is $l \sim 2.5 \times 10^{-27}$ and $\alpha=1 / 2$ [62]. The general behaviour of the cooling function was calculated and discussed by Sutherland and Dopita [63].

The projection on the sky of the plasma emissivity gives the $\mathrm{X}$-ray surface brightness: in order to constrain the physical parameters of the ICM, the observed surface brightness can be either geometrically deprojected or, more simply, fitted with a model obtained from an assumed distribution of the gas density.

4.3. Hydrostatic Models for ICM Distribution. From the expression for the sound speed $c_{s}^{2}=\gamma k T / \mu m_{p}$, where $\gamma=5 / 3$ for a monatomic gas, the time required for a sound wave in the ICM to cross a cluster is given by:

$$
t_{s} \approx 6.6 \times 10^{8}\left(\frac{T}{10^{8} \mathrm{~K}}\right)^{-1 / 2}\left(\frac{D}{1 \mathrm{Mpc}}\right) \mathrm{yr},
$$

where $D$ is the cluster diameter. Since this time is short compared to the likely age of a cluster (in first approximation assumed to be $\sim 10^{10} \mathrm{yr}$ ), the gas is generally thought to be in hydrostatic equilibrium in the gravitational potential of the cluster: $\nabla p=-\rho \nabla \phi$, where $p=\rho k T / \mu m_{p}$ is the gas pressure, $\rho$ is the gas density, and $\phi$ is the gravitational potential of the cluster. Under the assumptions that the ICM is locally homogeneous and the cluster is spherically symmetric, the hydrostatic equilibrium equation reduces to

$$
\frac{1}{\rho} \frac{d p}{d r}=-\frac{d \phi}{d r}=-\frac{G M(r)}{r^{2}},
$$

where $r$ is the radius from the cluster center and $M(r)$ is the total cluster mass within $r$. If the gas self-gravity is ignored, then the distribution of the ICM is determined by the cluster potential $\phi(r)$ and the temperature distribution of the gas $T(r)$, and (15) is a linear equation for the logarithm of the gas density. Under these assumptions, the gravitational mass $M_{\text {tot }}$ of a galaxy cluster can be written as:

$$
M_{\mathrm{tot}}(<r)=-\frac{k T r}{G \mu m_{p}}\left[\frac{d \ln \rho}{d \ln r}+\frac{d \ln T}{d \ln r}\right] .
$$

This expression is commonly used to estimate the gravitational mass of galaxy clusters and groups from X-ray observations, through the measurements of the radial profiles of temperature and density (e.g., [64-67]). However, we note that (16) neglects the contribution of possible additional, nonthermal pressure that, if present, should be included in the estimate of the total mass. In particular, recent results 
from numerical simulations indicate that the total mass of simulated clusters estimated through the X-ray approach is lower that the true one due to gas bulk motions (i.e., deviation from the hydrostatic equilibrium) and the complex thermal structure of the gas $[68,69]$. Possible observational biases in the derivation of $\mathrm{X}$-ray masses are also discussed in Piffaretti and Valdarnini [70].

4.3.1. The $\beta$-Model. Cavaliere and Fusco-Femiano [71] studied the X-ray emission by the hot plasma in galaxy clusters and developed a hydrostatic model based on the assumption that the gas and the galaxies are in equilibrium in the same gravitational potential $\phi$ (see, e.g., $[15,72]$ for a recent commentary on this model). By further assuming that the galaxy distribution is well described by King's approximation to the isothermal sphere [73], the expression for the ICM distribution may be written as [71]:

$$
\rho(r)=\rho_{0}\left[1+\left(\frac{r}{r_{\text {core }}}\right)^{2}\right]^{-(3 / 2) \beta},
$$

and the surface brightness profile observed at a projected radius $b, I(b)$, is in the form [71]:

$$
I(b)=I_{0}\left[1+\left(\frac{b}{r_{\text {core }}}\right)^{2}\right]^{1 / 2-3 \beta} .
$$

The parameter $\beta$ is defined as

$$
\beta=\frac{\sigma_{r}^{2}}{k T / \mu m_{p}},
$$

where $\sigma_{r}$ is the line-of-sight velocity dispersion and represents the ratio of specific kinetic energies of galaxies and gas.

This self-consistent isothermal model, called the " $\beta$ model," is widely used in the X-ray astronomy to parametrize the gas density profile in clusters of galaxies by fitting their surface brightness profile. One of the advantages of using a $\beta$ model to parameterize the observed X-ray surface brightness is that the total mass profiles can be recovered analytically and expressed by a simple formula:

$$
M_{\mathrm{tot}}(<r)=\frac{k r^{2}}{G \mu m_{p}}\left[\frac{3 \beta r T}{r^{2}+r_{c}^{2}}-\frac{d T}{d r}\right] .
$$

Equation (17) states that the gas density rises towards the cluster center. Since the bremsstrahlung and line emission depend on the square of the gas density (12), in the central regions of clusters the loss of energy by X-ray emission represents an important process for the thermal particles in the ICM. In particular, if the gas density reaches high enough values, large amounts of gas cool and flow into the centers of clusters, forming the so-called cooling flows. In cooling flow clusters, the single $\beta$-model is found to be a poor description of the entire surface brightness profile: a fit to the outer regions shows a strong excess in the center as compared to the model (see Section 4.4). Conversely, the centrally peaked emission is a strong indication of a cooling flow in relaxed cluster.
4.4. Cool Cores and Cooling Flow Problem. The X-rays emitted from clusters of galaxies represent a loss of energy of the ICM. The resultant cooling time is calculated as the time taken for the gas to radiate its enthalpy per unit volume $H_{v}$ using the instantaneous cooling rate at any temperature:

$$
t_{\mathrm{cool}} \approx \frac{H_{v}}{n_{e} n_{\mathrm{H}} \Lambda(T)}=\frac{\gamma}{\gamma-1} \frac{k T}{\mu X n_{e} \Lambda(T)},
$$

where $\gamma=5 / 3$ is the adiabatic index; $\mu \approx 0.6$ is the molecular weight; $X \approx 0.71$ is the hydrogen mass fraction; $\Lambda(T)$ is the cooling function. In the central region, the cooling rate of the ICM is sufficiently high that the particles lose their energy via radiation, as inferred from X-ray images of the cores of many clusters which show strongly peaked surface brightness distributions. The density of the gas then rises to maintain the pressure required to support the weight of the overlying gas in the rest of the cluster, causing a slow subsonic inflow of material towards the cluster center. This qualitative picture describes the physics of the process known as a cooling flow (see [2] for a review of the standard model, and [74] for a quantitative description of the evolution of cooling flows). The steady cooling flow is confined within the region in which $t_{\text {cool }}$ is less than the time for which the system has been relaxed (thus allowing the establishment of a cooling flow). This cooling region is delimited by the socalled cooling radius $r_{\text {cool, }}$, which is usually defined as the radius at which $t_{\text {cool }}$ is equal to the look-back time to $z=1$, that is, $\sim 7.7 \times 10^{9} \mathrm{yr}$ in the concordance cosmology. The fraction of clusters with a central surface excess with respect to a $\beta$-model, the so-called cool cores, is large. Cool core clusters are about $90 \%$ of X-ray-selected clusters with total mass $M_{\text {tot }} \lesssim 10^{14} M_{\odot}$, and about $50 \%$ of X-ray-selected clusters with total mass $M_{\text {tot }} \gtrsim 10^{14} M_{\odot}$ [75]. Cool cores are also characterized by strong enhancements in the central abundance (e.g., $[76,77])$ and declining temperature profiles toward the central region (e.g., [78-80]).

In the standard model, the "magnitude" of a cooling flow is measured from the amount of matter which crosses $r_{\text {cool }}$ and flows towards the center, that is, $\dot{M}$, the mass inflow rate. The mass inflow rate, due to cooling, can be estimated from the X-ray images by using the luminosity $L_{\text {cool }}$ associated with the cooling region and assuming that it is all due to the radiation of the total thermal energy of the gas plus the $p d V$ work done on the gas as it enters $r_{c}$ : $L_{\text {cool }}=d E / d t$, where $d E=d E_{\text {th }}+p d V=(\gamma / \gamma-1) p d V$, and $p d V=(\rho k T d V) /\left(\mu m_{p}\right)=(d M k T) /\left(\mu m_{p}\right)$, with $\gamma=5 / 3$. By substituting one obtains the expression for $L_{\text {cool }}$ :

$$
L_{\mathrm{cool}}=\frac{5}{2} \frac{\dot{M}}{\mu m_{p}} k T,
$$

where $T$ is the temperature of the gas at $r_{\text {cool }}$. $L_{\text {cool }}$ ranges from $\sim 10^{42}$ to $>10^{44} \mathrm{erg} \mathrm{s}^{-1}$ and generally represents $\sim 10 \%$ of the total cluster luminosity [2]. Value of $\dot{M} \sim 100 \mathrm{M}_{\odot} \mathrm{yr}^{-1}$ are fairly typical for cluster cooling flows.

However, the current generation of X-ray satellites, Chandra and XMM-Newton, has radically changed our understanding of cooling flow systems. Albeit confirming the existence of short cooling times, high densities and low 
temperatures in the cluster cores, the arrival of high-resolution X-ray spectral data has shown the absence or weakness of the soft X-ray line Fe XVII, indicating that the amount of gas cooling radiatively below about one-third of its original temperature is ten times less than expected (e.g., $[3,4,81]$ ). The lack of evidence for central gas cooling to very low temperatures at the rates predicted in the hot atmospheres of galaxy clusters and groups represents an open question which is often referred to as the so called "cooling flow problem" (see $[5,6,82]$ for reviews).

Historically, two main approaches were adopted to solve this problem. As the gas radiates but does not appear to cool, either the normal signatures of radiative cooling below 1-2 keV are somehow suppressed, or there must be an energy-injection mechanism into the ICM which compensates cooling. Different possibilities considered in the former hypothesis include absorption [3, 83], inhomogeneous metallicity $[83,84]$, and the emerging of the missing $\mathrm{X}$-ray luminosity in other bands, like ultraviolet, optical and infrared due to mixing with cooler gas/dust $[83,85,86]$. Proposed heating mechanisms in the context of the latter approach include, for example, processes associated with relativistic AGN outflows [87-93], electron thermal conduction from the outer regions of clusters [94-97], continuous subcluster merging [98], contribution of the gravitational potential of the cluster core [99], and feedback from intracluster supernovae [100]. Among all these, feedback by the central AGN appears to be the most promising solution.

\section{X-Ray Cavities and Shocks: AGN Feedback in Action}

It was already known in the early 90s that central dominant (cD) galaxies of cool core clusters have a high incidence of radio activity, showing the presence of central FR-I radio galaxies in $70 \%$ of the cases $[7,101,102]$. Their behaviour differs from that of quasar: in many low-accretion-rate AGNs almost all the released energy is channeled into jets because the density of the gas surrounding the black hole is not high enough for an efficient radiation (e.g., [103]). In fact, the importance of these objects has been underestimated for a long time due to their poor optical luminosity. The importance of radio galaxies in cool cores began to emerge after the discovery, with the X-ray satellite ROSAT, of deficits in the X-ray emission of the Perseus and Cygnus A clusters which are spatially coincident with regions of enhanced synchrotron emission $[104,105]$. With the advent of the new high-resolution X-ray observations performed with the current generations of X-ray telescopes, Chandra and XMMNewton, it became clear that the central radio sources have a profound, persistent effect on the ICM. In particular, Chandra images, which are obtained at the superb angular resolution of $\sim 1^{\prime \prime}$, showed that the Perseus and Cygnus A clusters are not isolated cases-indeed the central hot gas in many cool core systems is not smoothly distributed, but is instead cavitated on scales often approximately coincident with lobes of extended radio emission. These observations also reveal highly disturbed structures in the cores of many clusters, including shocks, ripples, and sharp density discontinuities. The comparison with radio images obtained at similar angular resolution has revealed that AGN jets are the cause of many of these disturbances. The most typical configuration is for jets from the central dominant elliptical of a cluster to extend outwards in a bipolar flow, inflating lobes of radio-emitting plasma. These lobes push aside the X-ray emitting gas of the cluster atmosphere, thus excavating depressions in the ICM which are detectable as apparent "cavities" in the X-ray images. The brightness depressions observed in X-rays, which are mostly found in pairs, are $\sim 20-40 \%$ below the level of the surrounding gas, consistently with the expected decrement along an empty bubble immersed in a $\beta$-model atmosphere [106-110]. The cavities are often surrounded by bright shells, or rims, which are typically found to be cooler than the ambient medium $[106-109,111]$. This is likely due to the compression of the central, low-entropy gas into the bright shell during the cavity rising and expansion into the hot atmosphere.

$\mathrm{X}$-ray cavities are present in $\gtrsim 70 \%$ of cool-core clusters [112], but this fraction could be underestimated due to the limitation of cavity detectability [113]. Identifying radio galaxies as a primary source of feedback in the hot atmospheres of galaxy clusters and groups has been one of the major achievements of the current generation of Xray observatories (for a comprehensive review see [6] and references therein). Well-studied examples of cavity clusters are Perseus (e.g., $[104,106,114,115])$, Hydra A (e.g., [4, 107, 116-120]), M 87 (e.g., [89, 121-125]), A 2052 (e.g., [108, 126-128]), RBS 797 (e.g., [111, 129-131]), A 133 (e.g., $[132,133])$, A 262 (e.g., $[126,134])$, and MS $0735+7421$ (e.g., $[135,136])$. In-depth analyses of individual objects, which are now numerous in the literature, combined with studies of cavity samples $[8,112,137-140]$ have enabled us to identify the global properties which are common among the cavities, thus shedding light of the feedback mechanism. The emerging picture is that bipolar outflows emanating from the BCG core inflate large bubbles while driving weak shocks, heat the ICM and induce a circulation of gas and metals on scales of several $100 \mathrm{~s} \mathrm{kpc}$. Weak shocks have been observed as ripple-like features in the ICM in the deepest X-ray images of Perseus and A 2052 [115, 128].

However, the differences between groups and clusters imply that the existing studies on cavities in clusters tell us little about how feedback operates in groups. With respect to rich galaxy clusters, the observation of cavities in galaxy groups and ellipticals is complicated by the lower X-ray surface brightness, which limit their detection in shallow Xray images. On the other hand, there are several examples of AGN-ICM interaction just a few tens Mpc away from us, which allow us to probe regions closer to the central black hole. In particular, low mass systems with cavities which now have deep Chandra images are M 84 [141, 142], NGC 4636 [143-145], NGC 5044 [146-149], HCG 62 [150153], and NGC 5846 [154]. Performing in-depth individual studies and sample studies of the lower-energy outbursts in these smaller systems is of major interest because the relationship between AGNs and hot gas can significantly influence galaxy evolution in the group environment, which 


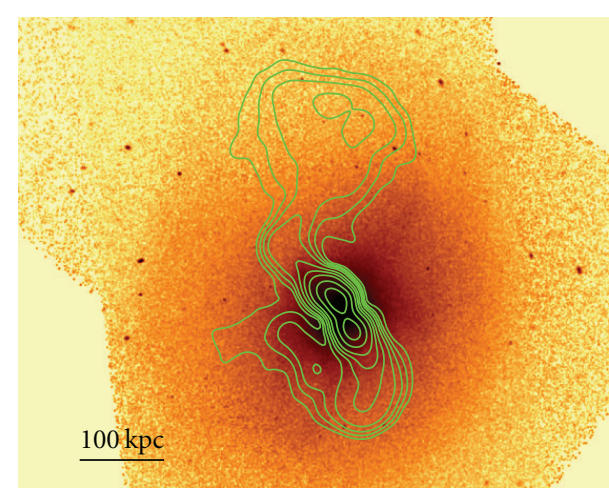

(a)

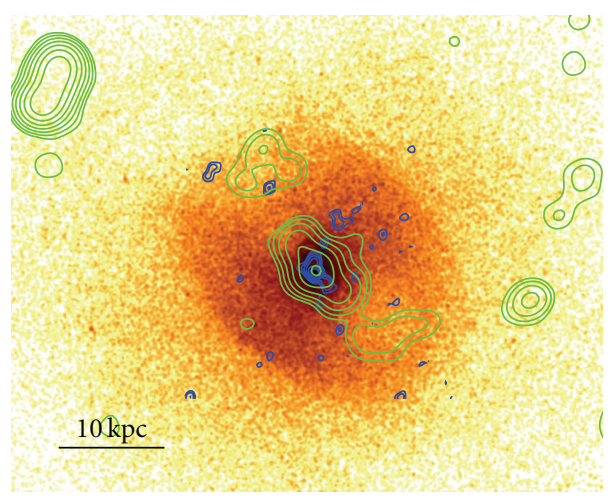

(c)

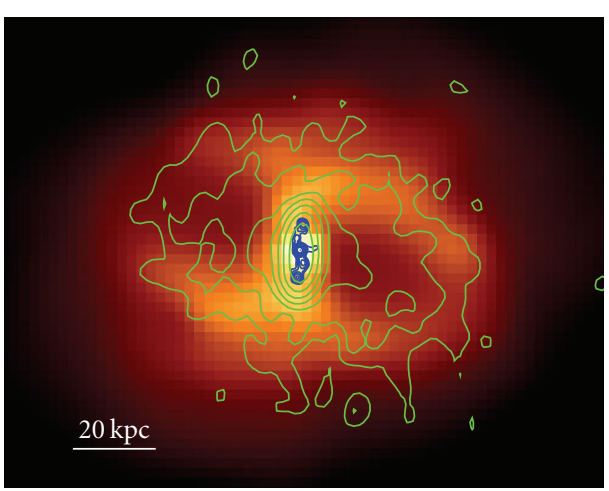

(b)

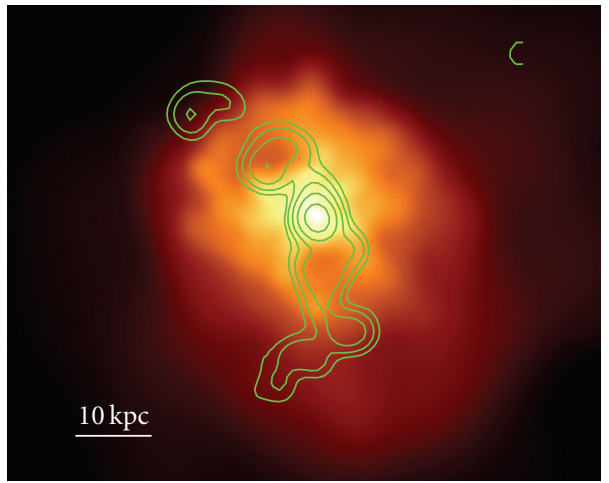

(d)

Figure 1: (a) The green contours outlining the $330 \mathrm{MHz}$ radio emission from Lane et al. [164] are overlaid onto the 0.5-7.5 keV Chandra image of the galaxy cluster Hydra A $(z=0.0538)$. The extended radio lobes fill a large-scale system of X-ray cavities and are surrounded by a "cocoon" shock. See also Sections 5.6 and 5.8. adapted from Nulsen et al. [116]. (b) Very Large Array (VLA) radio contours overlaid onto the 0.5-7.0 Chandra image of the galaxy cluster RBS $797(z=0.35)$. The subarsec resolution radio image shows the details of the innermost $4.8 \mathrm{GHz}$ radio jets (blue contours), which clearly point in a north-south direction. Remarkably, these inner jets are almost perpendicular to the axis of the $1.4 \mathrm{GHz}$ emission observed at $1^{\prime \prime}$ resolution (green contours), which is elongated in the northeast-southwest direction filling the X-ray cavities. Adapted from Gitti et al. [130]. (c) $0.3-2 \mathrm{keV}$ Chandra image of the galaxy group NGC 5813 $(z=0.0066)$ with $1.4 \mathrm{GHz}$ VLA (blue) and $235 \mathrm{MHz}$ Giant Metrewave Radio Telescope (green) radio contours overlaid. The image shows two pairs of cavities, plus an outer cavity to the northeast, two sharp edges to the northwest and southeast, and bright rims around the pair of inner cavities. Adapted from Randall et al. [157]. (d) $235 \mathrm{MHz}$ GMRT contours overlaid on the smoothed 0.5-2.0 keV Chandra image of the compact group HCG $62(z=0.0137)$. The radio source shows a disturbed morphology with inner lobes clearly filling the well-defined X-ray cavities, but with outer lobes having no associated X-ray cavities (see also Section 5.4). Adapted from Gitti et al. [153].

is the locus of the majority of galaxies in the Universe [155]. Due to the shallower group potential, the AGN outburst can have a large impact on the intragroup medium in terms of altering the thermal history and spatial distribution of the intragroup medium, as the mechanical output by radio AGN is of the same order of magnitude as the binding energy of groups [156]. Such investigations have been undertaken only recently for individual objects (e.g., NGC 5813: [157], AWM 4: $[158,159])$ and for group samples [9, 160-162], and are rapidly improving our understanding of these systems. However, this observational effort is still awaiting detailed theoretical work in order to corroborate the observational findings. Recent detailed simulations indicate that groups are not simply a scaled-down version of clusters, as there may be remarkable differences between how AGN feedback operates in galaxy group and in galaxy cluster environments [163]. In particular, AGN heating in groups seems to act through persistent, gentle injection of mechanical energy. On the other hand, in clusters there must be also the action of rare, powerful outbursts [163], although more extensive theoretical work is required to reach firm conclusions.

Examples of well-studied cavity systems in clusters and groups are shown in Figure 1.

5.1. Cavity Heating. The heating is thought to occur through the dissipation of the cavity enthalpy and through shocks driven by the AGN outburst. The energy required to create a cavity with pressure $p$ and volume $V$ is the sum of the $p V$ work done by the jet to displace the $\mathrm{X}$-ray emitting gas while it inflates the radio bubble, and the internal energy of the lobes, that is, the enthalpy given by:

$$
E_{\mathrm{cav}} \equiv H=E_{\mathrm{int}}+p V=\frac{\gamma}{\gamma-1} p V
$$

where $\gamma$ is the ratio of the specific heats of the cavity content. If the lobes are dominated by the magnetic field, by 
nonrelativistic gas, or by relativistic plasma, $H$ can vary from $2 p V$ and $4 p V$. In particular, typically it is assumed that the internal composition of the cavity is dominated by relativistic plasma, therefore $\gamma=4 / 3$ and $H=4 p V$. The product of pressure and volume can be estimated directly by X-ray observations through measurements of the cavity size and of the temperature and density of the surrounding ICM. A potential issue is represented by the uncertainties in the measurement of the cavity volume. The cavity size is usually estimated through a visual inspection of the X-ray images. This method is therefore dependent on the quality of the Xray data and also subject to the arbitrariness of the observer. The cavity size and geometry measured by different observers may vary significantly depending on the approach adopted, leading to differences between estimates of up to a factor of a few in $p V$ (e.g., $[131,153,165])$.

Systematic observational studies of samples of X-ray cavities show that their enthalpies measured from (23) lie between $\sim 10^{55} \mathrm{erg}$ (in ellipticals, groups, and poor clusters) and $\gtrsim 10^{61} \mathrm{erg}$ (in rich clusters). On the other hand, simulations indicate that $p V$ varies with time during the cavity evolution and may be an inaccurate measure of the total energy released [166, 167]. Cavity power estimates within a factor of two of the simulated values seem possible provided the inclination angle of the jets is known accurately [168]. Bearing this caveat in mind, when divided by the cavity age, $t_{\mathrm{cav}}$, the observational measurements give an estimate of the so-called "cavity power," $P_{\text {cav }}$. Since shocks are very difficult to detect and are currently known only in a few systems (e.g., Hydra A [116], MS 0735 + 7421 [135], HCG 62 [153], NGC 5813 [157]), for consistency, the usual approach in sample studies is that of considering only the cavity power. $P_{\text {cav }}$ thus provides a lower limit (and best-available gauge) to the true total mechanical power of the AGN, that is, the jet power: $P_{\text {jet }} \gtrsim P_{\text {cav }}=E_{\text {cav }} / t_{\text {cav }}$.

As proposed by Bîrzan et al. [137], the cavity age can be estimated in three ways: (1) by assuming that the cavity rises the hot gas atmosphere at the sound speed $c_{s}=\sqrt{\gamma k T / \mu m_{p}}$ in this case the cavity reaches the projected distance $R$ from the cluster center in the sound crossing time $t_{s}=R / c_{s}=$ $R / \sqrt{\gamma k T / \mu m_{p}}$; (2) by assuming that the cavity is buoyant and move outwards at the terminal velocity $v_{t}=\sqrt{2 g V / S C}$, where $g=G M_{<R} / R^{2}$ is the gravitational acceleration at the cavity position $R, V$ is the volume of the cavity, $S$ is the cross-section of the cavity, and $C=0.75$ is the drag coefficient [89]: in this case the cavity age is the buoyancy-time $t_{\text {buoy }} \sim R / \sqrt{2 g V / S C}$; (3) by considering the time required for gas to refill the displaced volume of the cavity as it rises: in this case the cavity age is estimated as $t_{\text {ref }} \sim 2 \sqrt{r / g}$, where $r$ is the radius of the cavity. Typically, the age estimates agree to within a factor of 2, with the buoyancy times lying in between the sound crossing time and the refill times. Most sample studies adopt the buoyancy time, which for typical values gives cavity ages of the order of few $10^{7} \mathrm{yr}$ (e.g., [139]).

5.2. The Relationship between Jet Power and $L_{\text {cool }}$. Once a cavity is detected, it is relatively simple to estimate its power from the measurements of $E_{\mathrm{cav}}$ and $t_{\mathrm{cav}}$ by applying (23). The cavity power, $P_{\mathrm{cav}}$, which is a measure of the energy injected into the hot gas by the AGN outburst, can then be compared directly with the gas luminosity inside the cooling radius, $L_{\text {cool }}$, which represents the luminosity that must be compensated for by heating to prevent cooling. The gas luminosity inside the cooling radius is estimated as the bolometric Xray luminosity derived from a deprojection spectral analysis. In Figure 2(a) is shown a quantitative comparison between $P_{\text {cav }}=4 p V / t_{\text {buoy }}$ and $L_{\text {cool }}$ calculated for the extended sample discussed in O'Sullivan et al. [165], who combined new data of 9 galaxy groups with the cluster sample of Bîrzan et al. [8] and with the elliptical sample of Cavagnolo et al. [169].

This plot follows those presented in Figure 2 of Bîrzan et al. [137] and in Figure 6 of Rafferty et al. [139]. As already noted by these authors, it is evident that the cavity power scales in proportion to the cooling X-ray luminosity, although with a big scatter. In general, it appears that the high mass (corresponding to high $L_{\text {cool }}$ ) systems need an average of $4 p V$ per cavity to counter cooling. On the other hand, if we recalculate $P_{\text {cav }}$ as $1 p V / t_{\text {buoy }}$ all the points in the plot will shift down by a factor 4 , and only the lower mass systems will still lie around the line $P_{\text {cav }}=L_{\text {cool }}$. These systems require $1 p V$ per cavity to offset cooling at the present time. A few low mass systems will even still be above the equality line, thus indicating that the total mechanical power of the AGN far exceeds the radiative losses and their atmospheres are being heated. Although this extended sample is not a complete sample and therefore is not representative of the whole population of clusters, groups, and ellipticals, it is interesting to consider the mean values of heating and cooling to see how they compare. We estimated a mean cooling power of $4.09 \times 10^{44} \mathrm{erg} \mathrm{s}^{-1}$, and a mean cavity power of $6.18 \times$ $10^{44} \mathrm{erg} \mathrm{s}^{-1}$. In order to quantify properly the contribution of AGN feedback, over the system lifetime, in the energetics of cooling flow, it is important to determine the "duty-cycle" of AGN. Many studies have attempted such calculation by adopting different approaches, for example, by considering the luminosity function of radio galaxies [170], the fraction of clusters that contain bubbles and cavities [112, 138, 171], the frequency of bubble required to produce sufficient heating [172], the prevalence of radio-loud AGN $[101,173]$. In particular, by considering the cavities as tracers of the feedback mechanism, that is, by assuming that the feedback is active and efficient as long as the cavities are visible, we can correct the mean cavity power by the fraction of cool core clusters with cavities, estimated by Dunn and Fabian [112] of the order of at least $70 \%$. The ratio of mean cavity power to cooling flow power is thus very close to unity. The mean values for the whole sample are only indicative and do not reflect the different behaviour of groups and ellipticals with respect to clusters. In particular, such mean powers miss the point that in order to counter cooling the low mass systems require outbursts with relatively less total energy, lower powers, and repeating more rapidly than high mass systems. This is supported by recent numerical simulations of galaxy groups which show that, in contrast to galaxy clusters, the AGN self-regulated feedback must act through a quasi-continuous gentle injection with subrelativistic outflows, rather 


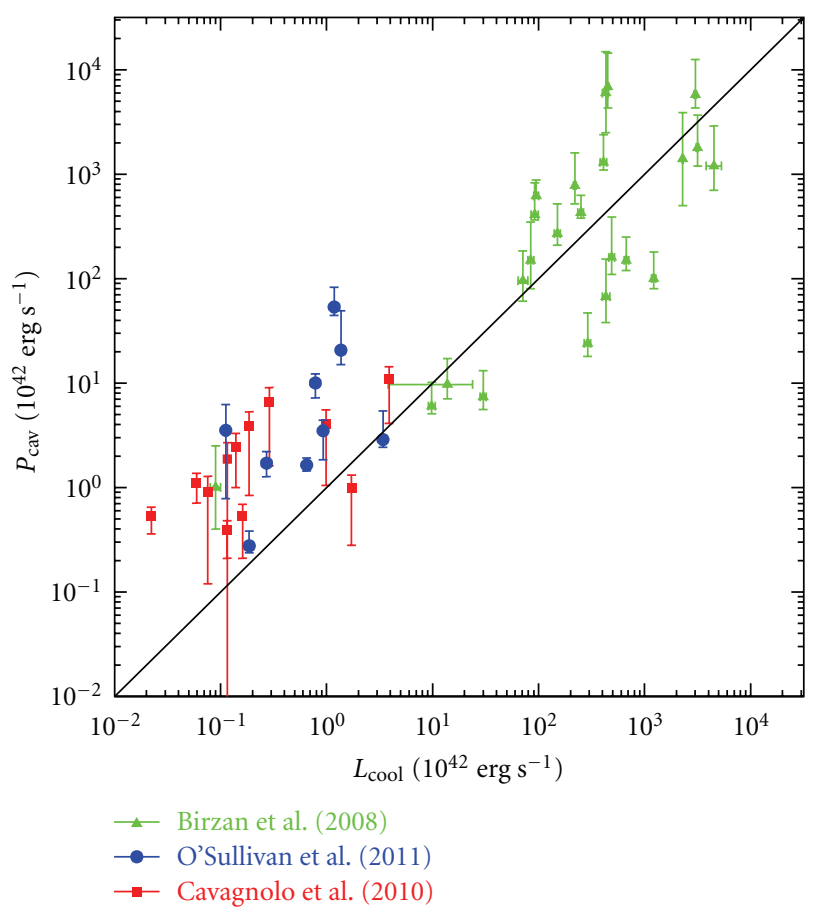

(a)

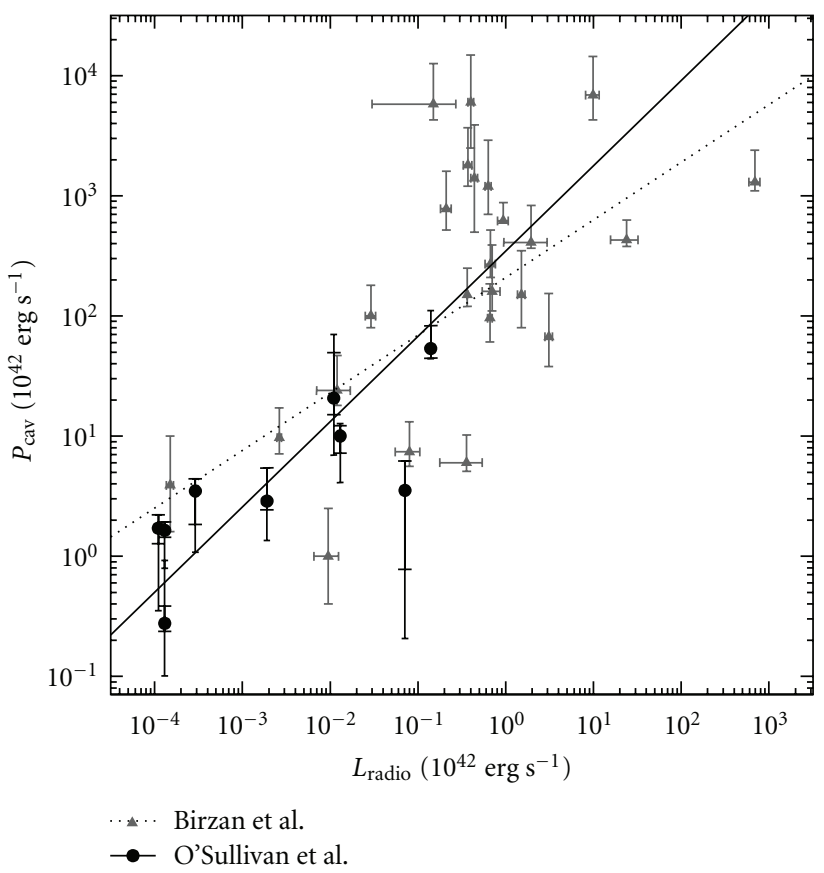

(b)

Figure 2: (a) Cavity power of the central AGN, $P_{\text {cav }}$, versus the X-ray luminosity of the ICM inside the cooling region, $L_{\text {cool }}$. The cavity power is calculated assuming $4 p V$ of energy per cavity and the buoyancy timescale. Different symbols denote systems in different samples presented in the literature: green triangles: Bîrzan et al. [8], red squares: Cavagnolo et al. [169], blue circles: O'Sullivan et al. [165]. 1 $\sigma$ uncertainties on cavity power are indicated by error bars (see [165] for details). The diagonal line denotes $P_{\text {cav }}=L_{\text {cool }}$. Credit O'Sullivan (private communication). (b) Cavity power of the central AGN, $P_{\text {cav }}$, versus integrated $10 \mathrm{MHz}-10 \mathrm{GHz}$ radio power, $L_{\text {radio }}$, for the systems in the sample of Bîrzan et al. (grey triangles) and the groups in the sample of O'Sullivan et al. (black circles). The solid fit line indicates the regression fit to the data points calculated by O'Sullivan et al. [165]. The dotted line indicates the relation found by Bîrzan et al. [8]. Adapted from O’Sullivan et al. [165].

than through rare and powerful episodes [163]. An attempt to produce more meaningful averages could be that of dividing the sample plotted in Figure 2(a) in two subsets. In fact, by doing this we find that the ratio of mean cavity power to cooling power for the groups and ellipticals is 7.94 (samples of Cavagnolo et al. [169] and O'Sullivan et al. [165]), compared to a ratio of 1.51 calculated for clusters only (sample of Bîrzan et al. [8]). If the duty cycle of low mass systems is the same as (or not lower than) high mass systems $[112,173]$, the relative ratio of heating to cooling appears to be a factor $\gtrsim 5$ higher in low mass systems. In other words, groups and ellipticals seem to have five times as much power available to counter cooling than rich clusters.

A study of a complete unbiased sample including both cool-core and non cool-core systems is necessary to derive definite constraints on the balance between heating and cooling. However, it seems plausible that the timeaveraged AGN feedback balances radiation losses of the ICM. Therefore, the general picture emerging from the observed trend between X-ray luminosity and bubble mechanical luminosity, together with the existence of short central cooling time, is that the AGN is fueled by a cooling flow that is itself regulated by feedback from the AGN. The basic idea of this AGN-cooling flow scenario is that a self-regulated equilibrium may be achieved, in which the radiative losses from the thermal ICM are balanced by mechanical heating from the central AGN over the system lifetime. Although this scenario is no longer in doubt, it is still not clear how heating can act preserving at the same time the observed temperature gradient and the cool core (e.g., [174]).

5.3. The Relationship between Jet Power and Radio Power. Studies of cavity samples allow to derive the relationship between the mechanical power and radio emission of AGN jets and lobes. Such a relationship is of great interest because it helps to understand the physics of AGN jets (e.g., $[165,175])$, and because it provides an estimate of the energy available from AGN based directly on the radio data (e.g., [101]), thus avoiding the problem of cavity detectability in shallow X-ray images. Bîrzan et al. [8] studied a sample dominated by galaxy clusters and derived the relation between cavity power and $327 \mathrm{MHz}$ radio power, as well as between cavity power and the integrated $10 \mathrm{MHz}-$ $10 \mathrm{GHz}$ radio luminosity, extending to lower frequencies their previous work [137]. While many of the observed Xray cavities are filled with $1.4 \mathrm{GHz}$ radio-emitting plasma, some are undetected at this high frequency and have been referred to as "ghost cavities." These may result from the aging of the relativistic particle population or may have been inflated by events which produced only particles of low 
energy. Examinations of radio images at multiple (and low) frequencies is particularly important as the progressive loss of particle energy causes higher frequency emission to fade fastest and the spectral index to steepen, so that evidence of a former AGN activity may be reflected only at low frequencies. The lack of $1.4 \mathrm{GHz}$ radio emission is observed more frequently in groups than in clusters, therefore lowfrequency radio observations are crucial for galaxy groups.

Giacintucci et al. [9] selected a compilation of 18 galaxy groups, based on the presence of signs of interaction between the hot gas and the central AGN, and observed both by the Giant Metrewave Radio Telescope (GMRT) at frequencies $\leq 610 \mathrm{MHz}$ and by Chandra and/or XMM-Newton. These authors found that nine of these groups have cavities clearly correlated with radio structures. By adding such systems to the Bîrzan et al. [8] sample, O'Sullivan et al. [165] examined the relations between jet mechanical power and radio power in a combined sample which includes the groups having the most reliable radio measurements currently available. In particular, the integrated $10 \mathrm{MHz}-10 \mathrm{GHz}$ radio luminosity estimated from the source spectral index is considered by these authors as a superior cavity power indicator compared to estimates at a single frequency, since it accounts for variations in spectral index between sources. Figure 2(b) shows the relationship between cavity power, $P_{\text {cav }}$, and the integrated radio luminosity, $L_{\text {radio }}$, for the combined sample. The best fitting power-law relationship is [165]

$$
\log P_{\text {cav }}=0.71( \pm 0.11) \log L_{\text {radio }}+2.54( \pm 0.21),
$$

where $P_{\text {cav }}$ and $L_{\text {radio }}$ are in units of $10^{42} \mathrm{erg} \mathrm{s}^{-1}$. See O'Sullivan et al. [165] for a detailed discussion of this relation.

5.4. Radio Lobe Composition. When the radio source is filling the cavities, it is possible to compare the radio pressure of the relativistic plasma internal to the lobes with the X-ray pressure of the surrounding thermal gas. The pressure of the hot gas is measured from the density and temperature derived from the X-ray data as $p \simeq 2 n_{e} k T$. The total pressure in a radio lobe is the sum of the magnetic pressure, $p_{B}$, and the total particle pressure, $p_{\text {part }}$, and can be written as:

$$
p_{\text {radio }}=p_{B}+p_{\text {part }}=\frac{B^{2}}{8 \pi}+\frac{1}{3} \frac{E_{\text {part }}}{f V}=\frac{B^{2}}{8 \pi}+\frac{1}{3} \frac{(1+k) E_{e}}{f V},
$$

where $B$ is the magnetic field, $k$ is the ratio of the energy in protons to that in electrons $\left(E_{e}\right), V$ is the volume of the radio lobe, and $f$ is the volume filling factor of the relativistic plasma. Using the expression for $E_{e}$ given in Pacholczyk [176], (25) determines the lobe pressure in terms of the magnetic field strength and the factor $k / f$, once the volume $V$ of the radio lobe is known. This calculation is usually performed under the widely adopted minimum energy conditions, in which the relativistic plasma is in equipartition with the magnetic field $\left(B_{\text {eq }}\right)$. Further assumptions usually made in literature are $f=1$ and $k=0$ or $k=1$. A volume filling factor of 1 indicates that the lobes are empty of thermal gas, which is a reasonable hypothesis when they are observed to be spatially coincident with X-ray cavities. The assumption $k=1$ implies that half of the energy in particles is in the form of nonradiating particles, as in an electron-proton jet, whereas $k=0$ would indicate an electron-positron jet. We stress that the uncertainties in the calculation of $B_{\mathrm{eq}}$ and $p_{\text {radio }}$ come from the values of $k$ and $f$, which are still largely unknown. Conversely, it is possible to constrain the ratio $k / f$ by assuming pressure balance (see below).

For historical reasons the frequency band adopted to calculate the standard equipartition field is $\nu_{1}=10 \mathrm{MHz}-$ $v_{2}=100 \mathrm{GHz}$, that is, roughly the frequency range observable with radio telescopes. From a physical point of view, the adoption of this frequency band in the calculation of the minimum energy is equivalent to the assumption that only electrons emitting between $10 \mathrm{MHz}-100 \mathrm{GHz}$, that is, with energy between $\gamma_{\min } \propto\left(\nu_{1} / B_{\mathrm{eq}}\right)^{1 / 2}$ and $\gamma_{\max } \propto\left(\nu_{2} / B_{\mathrm{eq}}\right)^{1 / 2}$ are present in the radio source. This approach neglects the contribution of the electrons emitting below $10 \mathrm{MHz}$ and, as a more serious bias, in radio sources with different $B_{\text {eq }}$ selects different energy bands of the electron population because the energy of the electrons which emit synchrotron radiation at a given frequency depends on the magnetic field intensity [177]. A more consistent approach is to calculate the minimum energy conditions, in which $B_{\mathrm{eq}}$ does not depend on the emitted frequency band but directly on the low-energy cutoff of the electron spectrum (typically assumed to be $\gamma_{\min }=100$ ). These so-called "revised" equipartition conditions select also the contribution to the energetics due to the low-energy electrons [178].

It is typically found that in cavity systems the X-ray pressure is more than one order of magnitude higher than the radio pressure (e.g., $[108,153,179,180])$. It is also found that with revised equipartition the cavities are closer to pressure balance than they are with standard equipartition (e.g., $[153,165])$. Vice versa, by assuming that the lobes are in pressure equilibrium with the ambient gas it is possible to constrain the particle content within the radio lobes $[8,179$, 181]. In particular, one can determine the ratio $k_{\mathrm{bal}} / f$ that is required to achieve pressure balance under revised equipartition conditions. Several studies of the energetics and particle content of the radio lobes in cooling cores have found high values of $k_{\mathrm{bal}} / f$, up to several thousands (with standard equipartition) for active bubbles (e.g., $[8,138]$ ), suggesting that a large fraction of energy must be in nonradiating particles if $f$ is close to unity. On the other hand, the pressure imbalance found in the lobes of FR-I radio galaxies in a sample of galaxy groups appears to be linked to the radiosource morphology, that is, "plumed" sources typically have larger pressure deficits than "bridged" sources where the jets are embedded in the lobes [180]. The authors interpret this result as evidence that plumed sources have a higher entrainment rate due to the larger fraction of the jet surface which is in direct contact with the external medium, leading to an increase in $k / f$. Although the classification into bridged and plumed morphologies may not directly apply to radio sources at the center of cool core systems, typically having amorphous structures, this picture is consistent with the results of Dunn et al. [182] who argue that the large pressure imbalance observed in radio bubbles as those of the Perseus 
cluster is more likely to be due to entrainment rather than a relativistic proton population. Recent studies show lobes having no associated X-ray cavities (e.g., [153, 165]). Assuming their detection is not limited by the sensitivity of the current Chandra images, this suggests the possibility of mixing between ambient gas and radio plasma in the lobes. Therefore the $k_{\mathrm{bal}} / f>0$ values measured in such lobes is likely the results of entrainment of thermal gas through the hot gas atmosphere rather than an evidence of heavy jets ejected from the AGN.

5.5. Radio Mini-Halos. In some cases, the powerful radio galaxies at the center of cool-core clusters are surrounded by diffuse radio emission on scales $\sim 200-500 \mathrm{kpc}$ having steep radio spectra $\left(\alpha>1 ; S_{\nu} \propto v^{-\alpha}\right)$. These radio sources, generally referred to as "radio mini-halos," are synchrotron emission from $\mathrm{GeV}$ electrons diffusing through $\mu G$ magnetic fields. Although the central radio galaxy is the obvious candidate for the injection of the population of relativistic electrons, mini-halos do appear quite different from the extended lobes maintained by AGN, therefore their radio emission proves that magnetic fields permeate the ICM and at the same time may be indicative of the presence of diffuse relativistic electrons. In particular, due to the fact that the radiative lifetime of radio-emitting electrons $\left(\sim 10^{8} \mathrm{yr}\right)$ is much shorter than any reasonable transport time over the cluster scale, the relativistic electrons responsible for the extended radio emission from mini-halos need be continuously reenergized by various mechanisms associated with turbulence in the ICM (reaccelerated primary electrons), or freshly injected on a cluster-wide scale (e.g., as a result of the decay of charged pions produced in hadronic collisions, secondary electrons). Gitti et al. [187] developed a theoretical model which accounts for the origin of radio mini-halos as related to electron reacceleration by magnetohydrodynamic (MHD) turbulence, which is amplified by compression in the cool cores. In this model, the necessary energetics to power radio mini-halos is supplied by the cooling flow process itself, through the compressional work done on the ICM and the frozen-in magnetic field. The successful application of this model to two cool core clusters (Perseus: [187] and A 2626: [183]) has given support to a primary origin of the relativistic electrons radiating in radio mini-halos.

Radio mini-halos are rare, with only about a dozen objects known so far. Gitti et al. [183] selected an initial sample of five mini-halo clusters based on the presence of both a cool core and a diffuse, amorphous radio emission with no direct association with the central radio source: Perseus [188], A 2626 [183, 189], A 2142 [190], PKS 0745191 [191], and A 2390 [192]. In these clusters the size of the diffuse radio emission is comparable to that of the cooling region. These criteria are now typically adopted to identify mini-halos. However, the classification of a radio source as a mini-halo is not trivial: their detection is complicated by the fact that the diffuse, low surface brightness emission needs to be separated from the strong radio emission of the central radio galaxy. Furthermore, the criteria adopted to define mini-halos are somehow arbitrary (e.g., total size, morphology, presence of cool core) and some identifications are still controversial. This said, new detections of radio mini-halos have recently been claimed in the galaxy clusters RX J1347.5-1145 [184], Z 7160 [193], A 1835 [194], A 2029 [194], Ophiuchus [194, 195], RXC J1504.1-0248 [186], and RBS 797 (Figure 3(a), see also [111]).

Radio mini-halos are still poorly understood sources. Although secondary electron models have been proposed to explain the presence of their persistent, diffuse radio emission on large-scale in the ICM (e.g., [196, 197]), a primary origin of radio mini-halos is now favored by recent statistical studies [198] and by the observed trend between the radio power of mini-halos and the maximum power of cooling flows (see Figure 3(b)). This indicates a direct connection between cooling flows and radio mini-halos, that is, the most powerful radio mini-halos are associated with the most massive cooling flows, as expected in the framework of the Gitti et al.'s [187] model. However, the origin of the turbulence necessary to trigger the electron reacceleration is still debated. The signatures of minor dynamical activity have recently been detected in some mini-halo clusters, thus suggesting that additional or alternative turbulent energy for the reacceleration may be provided by minor mergers [184, 198] and related gas sloshing mechanism in cool core clusters $[199,200]$. Given the prevalence of mini-halos in clusters with X-ray cavities, another attractive possibility is that the turbulent energy is provided by a small fraction of the energy released by the bubbles rising from the central AGN (as suggested by [198]). Needless to say, a larger mini-halo sample as well as further theoretical investigations are necessary to reach a better understanding of this class of sources.

5.6. Weak Shocks and Giant Cavities. In addition to the cavity enthalpy, shocks driven by the AGN outburst may contain a large fraction of the energy released, thus working to heat the ICM. Such shocks have been long predicted by numerical simulations [201-203] but are difficult to detect since they are relatively weak (with Mach numbers $\sim 1-2$ ) and are seen in projection against the cooler, brighter gas in cluster cores. We also note that to establish these surface brightness discontinuities as shocks one must measure an increase in temperature in the so-called "postshock region," as the ICM is heated by the passage of the shock. Usually the existing images are too shallow to rule out, for example, the possibility that these features are cold front edges, due to gas sloshing (e.g., [19]). Besides a very few examples of strong shocks (e.g., Centaurus A, with Mach number 8, [204, 205]), only recently elliptical surface brightness edges, consistent with arising from weak shock fronts driven by the cavities as they initially expanded, have become to emerge in deep Chandra exposures of bright clusters and groups. Beautiful examples of cocoon shocks are visible in the Hydra A cluster [116, 135] and in the NGC 5813 group [157], see left panels of Figure 1.

The recent discovery of giant cavities and associated large-scale shocks in three clusters (MS $0735+7421$ [135], Hercules A [206], Hydra A [116]) has shown that AGN outbursts not only can affect the central regions, but also have an impact on cluster-wide scales possibly affecting the global properties of the ICM and the cluster scaling relations. 


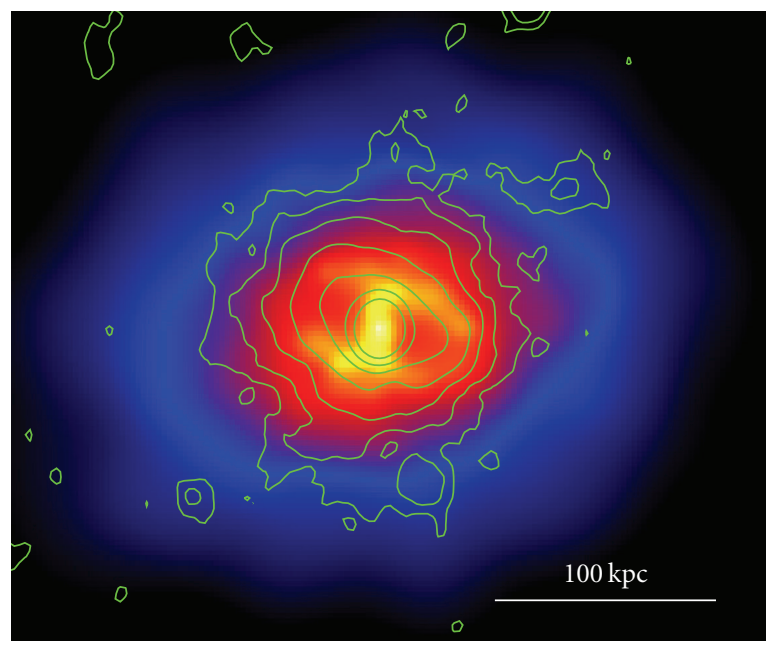

(a)

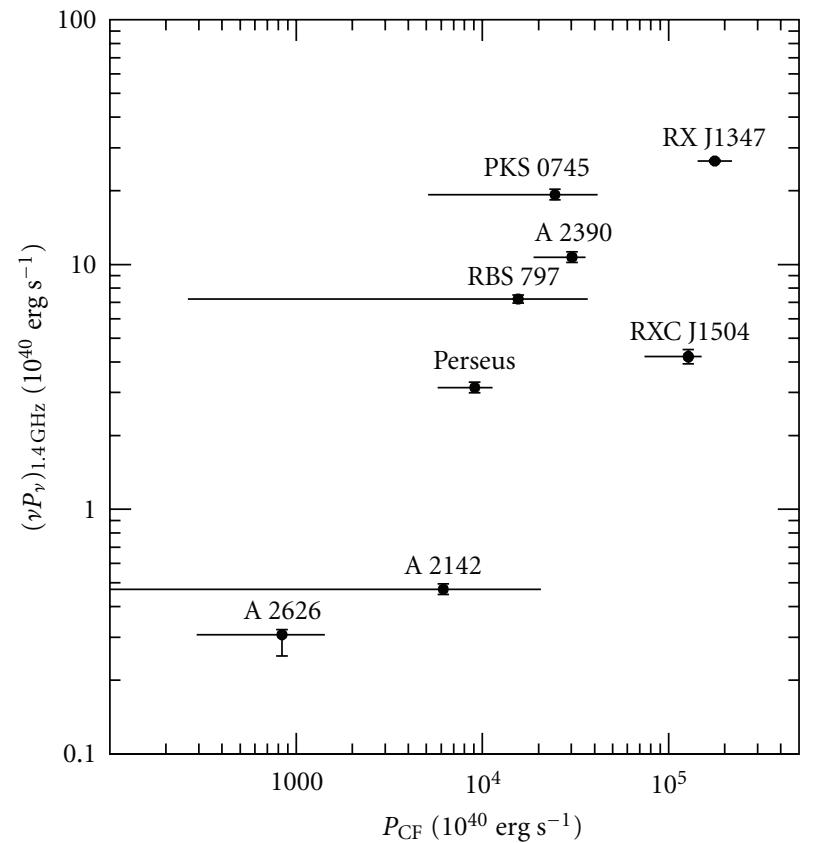

(b)

Figure 3: (a) 1.4 GHz VLA radio contours (obtained by combining observations in A-, B-, and C-array configurations) overlaid onto the smoothed $0.5-2.0 \mathrm{keV}$ Chandra X-ray image of the galaxy cluster RBS 797. The combined radio map has a resolution of $3^{\prime \prime}$, and is able to reveal the morphology of the central radio source, showing its elongation in the cavity direction (see Figure 1(b)), without losing sensitivity at the larger scales. In particular, the extended radio emission is detected out to $\sim 90 \mathrm{kpc}$. By subtracting the contribution of the central nuclear source, the residual flux density of the diffuse radio emission is $\simeq 11.5 \pm 0.6 \mathrm{mJy}$, indicating the likely presence of a radio minihalo. (b) Integrated radio power at $1.4 \mathrm{GHz},\left[\nu P_{\nu}\right]_{1.4 \mathrm{GHz}}$, versus cooling flow power, $P_{\mathrm{CF}}=\dot{M} k T / \mu m_{p}$, for the mini-halo clusters which have relevant X-ray and radio data available (data from $[111,183-186]$ ).

In particular, the giant cavities discovered in the galaxy cluster MS $0735+7421$ have a diameter of about $200 \mathrm{kpc}$ each. The large volume of the cavities implies a huge cavity power: this large-scale outburst is the most powerful known so far, releasing upward of $10^{61} \mathrm{erg}$ into the ICM and heating the gas beyond the cooling region $[135,136]$. The new, deep Chandra image has confirmed the presence of a weak (Mach number $\sim 1.3$ ) cocoon shock surrounding the cavity system (Figure 4).

This new development may have significant consequences for several fundamental problems in astrophysics. As seen in Section 2, the observed relation between X-ray luminosity and gas temperature in clusters is steeper than expected if cluster growth were governed by gravity alone. This steepening is best explained by the addition of heat to the ICM and is therefore considered the main manifestation of nongravitational heating. The discovery of giant cavities has indicated that powerful AGN outbursts occurring at late times may contribute a significant fraction of the extra nongravitational energy. As mentioned above, this additional heating supplied by AGN could also induce the suppression of the gas cooling in massive galaxies required to explain the exponential turnover at the bright end of the luminosity function of galaxies (e.g., [11]). This would indicate a common solution for the two major heating problems associated with the ICM: those of cooling flow and galaxy formation. In the case of MS $0735+7421$, the driving energy of the shock as determined using a spherical hydrodynamic model is $E_{s} \approx 5.7 \times 10^{61} \mathrm{erg}$ [135]. As estimated by Gitti et al. [136], the AGN outburst in this cluster is heating the gas mass within $1 \mathrm{Mpc}\left(\sim 7.7 \times 10^{13} \mathrm{M}_{\odot}\right)$ at the level of about $1 / 4 \mathrm{keV}$ per particle, and the heating level increases to $\sim 0.6 \mathrm{keV}$ per particle when considering the gas mass within $r_{2500}$. This is a substantial fraction of the $1-3 \mathrm{keV}$ per particle of excess energy required to heat the cluster [207]. Only a few outbursts of this magnitude erupting over the life of a cluster would be required to heat it. By contrast, MS $0735+7421$ is found to be a factor $\sim 2$ more luminous than expected from its average temperature on the basis of the observed $L-T$ relation for galaxy clusters [136]. Based on the data presented in Gitti et al. [120], we found a similar result for the giant cavity cluster Hydra A (Figure 5(a)). Although caution should be taken in drawing general conclusions from the study of only a few objects, this indicates that flux limited samples of distant X-ray clusters may be biased in favor of detecting clusters with energetic AGN outbursts. We also note that powerful AGN outbursts may have a dramatic effect on the gas mass fraction measurements, due to an overestimate of the gas density [136]. The observed departure of MS 0735+ 7421 and Hydra A from the mean $L-T$ relation is in apparent contradiction with the argument above that heat should steep the $L-T$ 


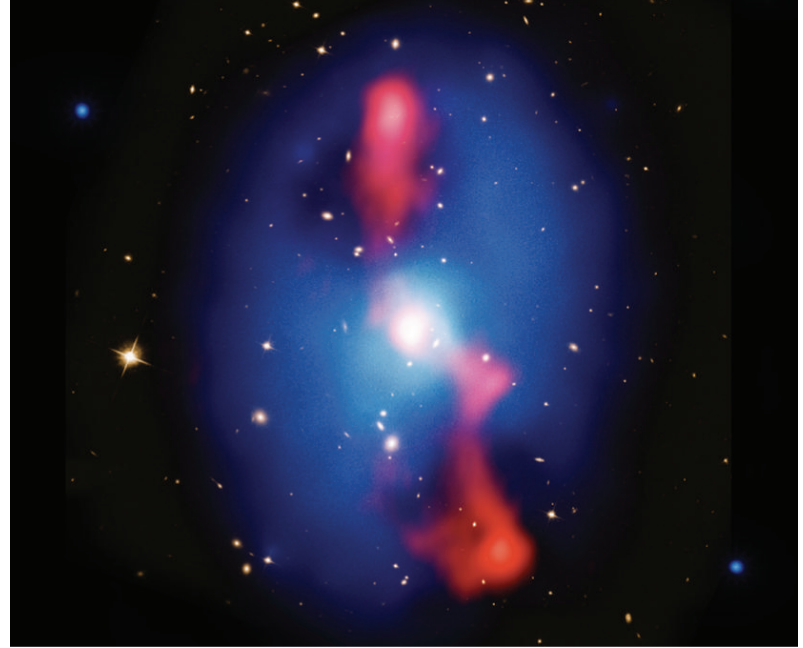

Figure 4: Deep 500 ks Chandra X-ray image (blue) and Very Large Array $330 \mathrm{MHz}$ radio image (red) superposed with the Hubble Space Telescope visual image of the galaxy cluster MS $0735+7421$. The giant X-ray cavities, filled with radio emission, are surrounded by a cocoon shock clearly visible in the Chandra image as an elliptical edge. The box is roughly $800 \mathrm{kpc}$ by $800 \mathrm{kpc}$.

relation, as also indicated by recent numerical simulations (e.g., [208]). However, we stress that the observed $L-T$ relation is highly dependent on the definition of the characteristic temperature, that is, for a fixed luminosity the position of each point in the plot may vary significantly depending on the choice of the method adopted to measure the average emission-weighted temperature for each cluster. Furthermore, the possibility of building a consistent $L-T$ scaling relation from a sample of clusters relies on the capability to correct both the temperature and the luminosity measurements for the effects of the central cooling flow in a consistent manner for the whole sample. This may not be trivial as the physical conditions can vary significantly from case to case. For example, the commonly adopted method of excluding the central $70 \mathrm{kpc}$ is found to have some drawback for giant cavity systems as the cooling region and the effect of AGN feedback extend beyond this radius (see discussion in [136]).

On the other hand, based on a study of XMM-Newton data, Gitti et al. [136] have shown that the energetic outburst in MS $0735+7421$ does not cause dramatic instantaneous departures from the average properties of the ICM because it has not had a measurable impact on the large-scale temperature profile, which is in fact consistent within the scatter of the profiles observed in relaxed cluster [79]. We recently found a similar result (Figure 5(b)) in the Hydra A cluster, although a sort of "plateau" standing below the typical profile in the range of radius $\sim 0.05-0.1 r_{\text {vir }}$ indicates the presence of cooler gas (see Section 5.8). In general, these results suggest that there cannot have been many previous outbursts of high magnitude in these clusters, otherwise the total energy added to the ICM outside the cooling region should have had a marked effect. Studies of cavity samples found that the prevalence of outbursts as energetic as $10^{61} \mathrm{erg}$ is 3 (namely, MS $0735+7421$, Hercules A and Hydra A) over 30 [139]. If, as it appears from our in-depth studies of MS $0735+7421$ and Hydra A, such powerful outbursts are rare in individual clusters, their occurrence in $\sim 10 \%$ of known cases hence requires that they occur a similar fraction of time in most cooling flow clusters. This picture is consistent with the model proposed by Nipoti and Binney [170], in which the AGN activity is strongly variable with time and all systems occasionally experience powerful outbursts.

5.7. SMBH Growth. AGN are powered by the release of gravitational binding energy from accretion onto massive black holes [211, 212]. The matter that reaches the black hole converts it binding energy efficiently into AGN power as $P_{\text {jet }}=\epsilon \dot{M} c^{2}$, where $\epsilon \sim 0.1-0.4$ depending on the spin of the black hole. Rapidly spinning black holes with spin parameters approaching unity are most efficient due to the smaller radius of the innermost stable circular orbit. The form of energy that is released depends on several factors including the accretion rate, the mass of the black hole, the structure of the accretion flow, and the spin of the black hole (see [213] for a review). When black hole accretion approaches the Eddington rate, the binding energy is emitted thermally from an optically thick, geometrically thin accretion disk that is morphologically classified as a quasar or Seyfert galaxy. When the accretion rate drops below a few percent of the Eddington rate a radiatively inefficient AGN is formed (i.e., an ADAF), and the energy is released primarily in the form of mechanical energy associated with a radio jet. Accretion rates in bright AGN can be estimated using the radiation emitted from the nucleus that directly (e.g., UV or X-ray emission) or indirectly (e.g., nebular or far-IR emission) trace the energetic output from the accretion disk (e.g., [214]). However, despite having mechanically powerful radio AGN [137], brightest cluster galaxies (BCGs) rarely show strong X-ray and UV emission emerging from their nuclei [215], implying that their accretion rates generally lie below a few percent of their Eddington rate.

Rafferty et al. [139] estimated the accretion rates in a sample of BCGs in clusters with prominent X-ray cavities and found this to be the case. They estimated the accretion rates using the measured output power based on the $p V$ work done by the cavities divided by their buoyancy ages. Using this approach, Rafferty et al. [139] found that supermassive black holes centred in cool cores are growing at a rate of $\sim 0.1 M_{\odot} \mathrm{yr}^{-1}$. In rare instances such as the powerful AGN in MS 0735 + 7421, [135, 136, 216], the accretion rate exceeds $1 M_{\odot} \mathrm{yr}^{-1}$. Assuming black hole masses that are consistent with the values expected from their stellar luminosities and velocity dispersions, the accretion rates are consistent with being currently at most a few percent of Eddington. If AGN feedback in BCGs quenches cooling flows over the lifetimes of clusters, their black holes may be more massive than predicted by the $M_{\mathrm{BH}}-\sigma$ relation.

5.8. Further Evidence for Mechanical Feedback. As we have seen in Section 5.2, it is now widely accepted that the AGN in the cool cores can reheat the ICM. Although this is certainly its main impact, AGN feedback is likely to have other 


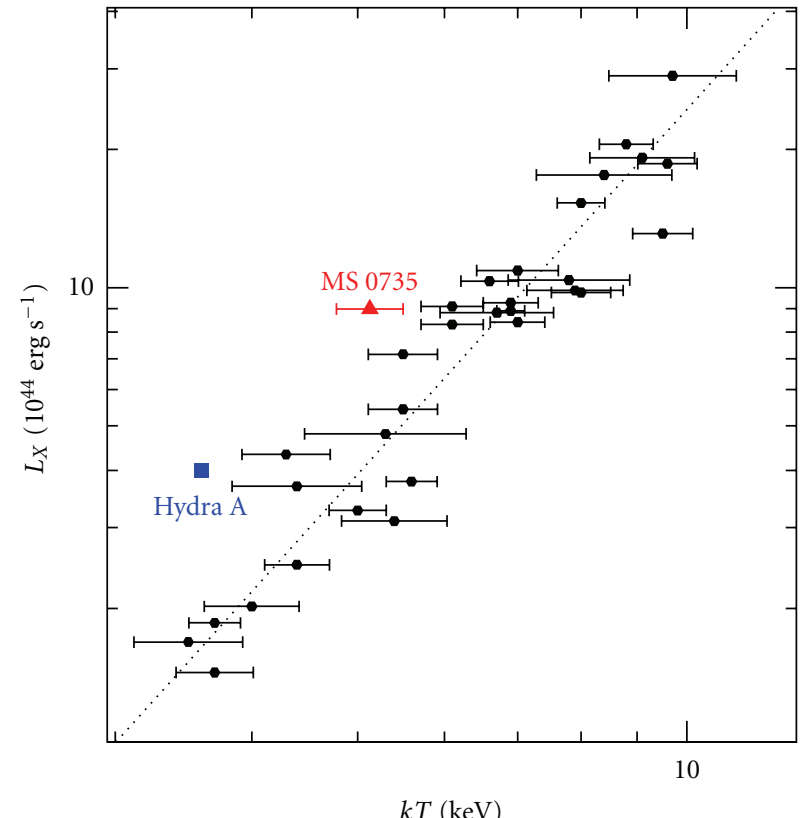

(a)

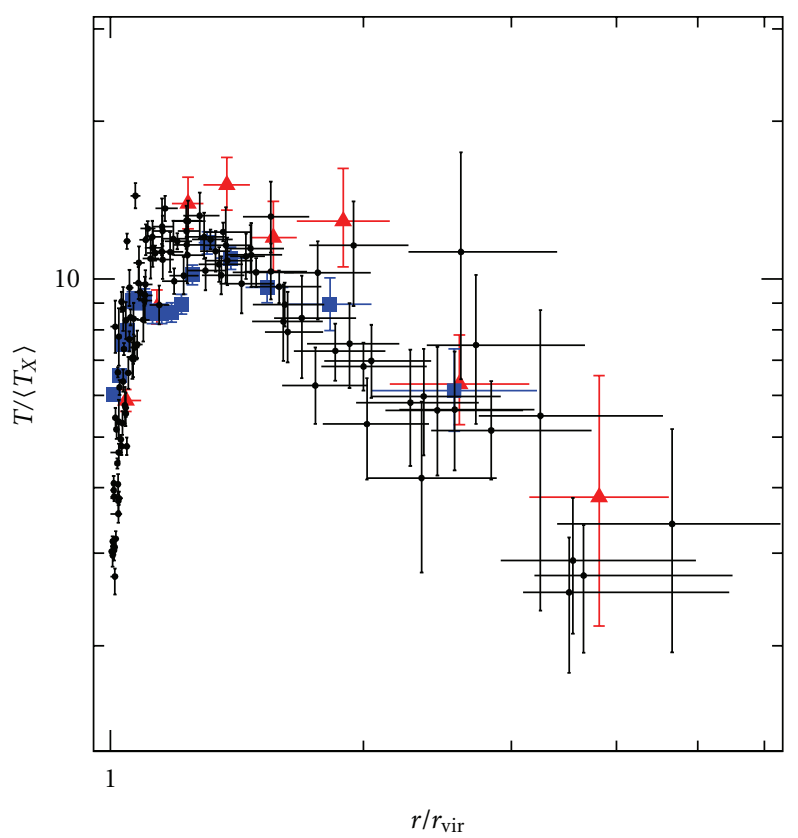

(b)

Figure 5: (a) Bolometric X-ray luminosities corrected for the effect of cooling flow in the central $\sim 70$ kpc versus emission-weighted temperatures derived excluding cooling flow components, from Markevitch [25]. The dashed line is the best-fit power law to the sample: $L_{X}=6.35 \cdot(\mathrm{kT} / 6 \mathrm{keV})^{2.64} \times 10^{44} \mathrm{erg} \mathrm{s}^{-1}$. The red triangle and the blue square represent the measurements from the XMM-Newton and Chandra data of the giant cavity clusters MS $0735+7421$ [209] and Hydra A, respectively, corrected consistently with the method adopted by Markevitch [25]. (b) Temperature profiles measured for a sample of relaxed clusters presented by Vikhlinin et al. [79]. The temperatures are scaled to the cluster emission-weighted temperature excluding the central $70 \mathrm{kpc}$ regions. The profiles for all clusters are projected and scaled in radial units of the virial radius $r_{\text {vir }}$, estimated from the relation $r_{\text {vir }}=2.74 \mathrm{Mpc} \sqrt{\left\langle T_{X}\right\rangle / 10 \mathrm{keV}}$ [210]. Overlaid are the cooling flow corrected, scaled temperature profiles of the giant cavity clusters MS 0735+ 7421 (red triangles, Gitti et al. [136]) and Hydra A (blue squares, Gitti et al. [120]).

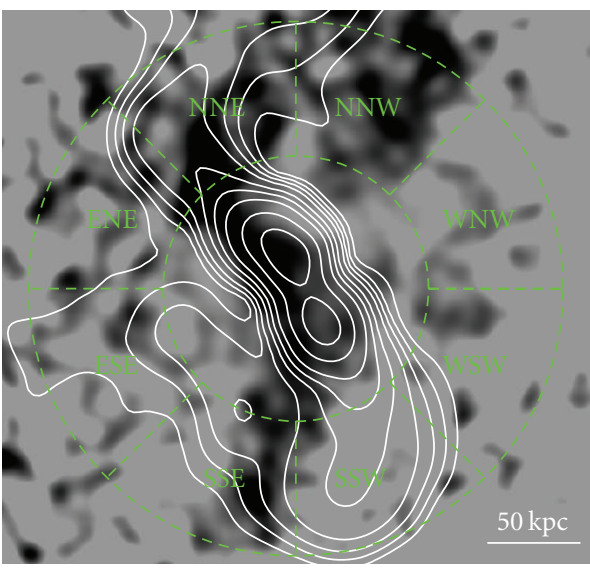

(a)

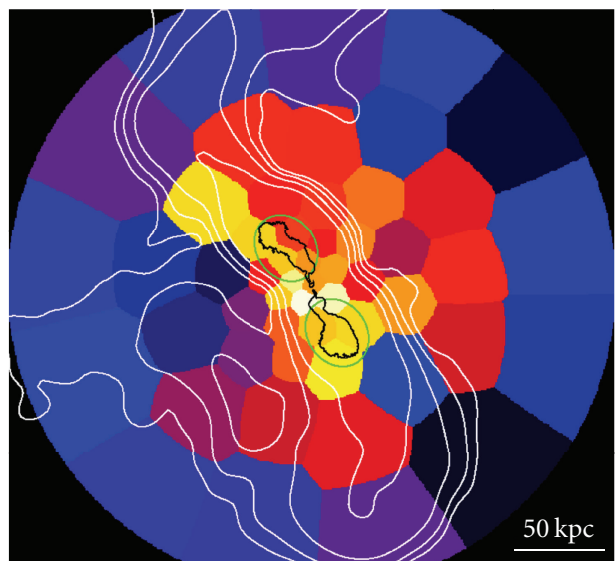

(b)

FIgURE 6: (a) High-contrast hardness ratio map of the galaxy cluster Hydra A obtained by dividing a $1.5-7.5 \mathrm{keV}$ image by a $0.3-1.5 \mathrm{keV}$ image. Regions in black are indicative of low temperature gas, indicating the presence of low-entropy filaments. Overlaid in green are the sectors used to study the spectral properties of the cool gas (located between radii $\sim 70-150 \mathrm{kpc}$ ). Gitti et al. [120] extracted the spectra in these sectors and compared two different spectral models: a single-temperature plasma " $1 \mathrm{~T}$ " model, and a " $2 \mathrm{~T}$ " model which includes a second thermal emission component. The F statistics for the spectral fitting improvement over the single-phase model indicate that the addition of a second thermal component is most significant in sectors SSE, NNW, NNE, and ENE, thus providing evidence for the presence of multiphase gas in agreement with the hardness ratio map. Adapted from Gitti et al. [120]. (b) Metallicity map showing the central $5^{\prime} \times 5^{\prime}$ of Hydra A. Brighter regions represent a higher metallicity. The color scale of the metallicity $Z$ (in solar units) is as follows: white: $Z \geq 0.75$, yellow: $Z=0.65-0.75$, orange: $Z=0.55-0.65$, red: $Z=0.45-0.55$, blue: $Z=0.3-0.45$, and black: $Z \leq 0.3$. The $1.4 \mathrm{GHz}$ radio emission is shown by the black contours. The green elliptical regions indicate the position of the inner cavities. Adapted from Kirkpatrick et al. [119]. In both panels are overlaid the white contours outlining the $330 \mathrm{MHz}$ radio emission from Lane et al. [164]. 
important effects on the ICM. Gitti et al. [120] have recently investigated this point by performing an in-depth study of the galaxy cluster Hydra A, which harbors a well-known, large-scale system of X-ray cavities embedded in a "cocoon" shock surrounding the central, powerful radio source ([107, 116], see Figure 1(a)). By means of a detailed spectral analysis of the deep ( $240 \mathrm{ks})$ Chandra observations, Gitti et al. [120] found indication of the presence of multiphase gas along soft filaments seen in the hardness ratio map (Figure 6(a)). Interestingly, such filaments are almost spatially coincident with the radio lobes of the powerful central radio source. The cooler gas has a significant impact on the radial temperature profile of the cluster, creating a sort of "plateau" which departs from the typical profile (blue squares in Figure 5(b)). In fact, the scaled temperature profile of Hydra A measured after masking the filaments is found to agree well with the general shape of the temperature profiles observed for relaxed clusters, thus providing a confirmation that these filaments contain cool gas [120]. By performing a spectral deprojection analysis of an absorbed 2-temperature component model, Gitti et al. [120] found evidence that $\sim 10^{11} M_{\odot}$ of low-entropy material has moved upward from the central $30 \mathrm{kpc}$ to the observed current position of $75-150 \mathrm{kpc}$, likely due to some form of entrainment or dredge up by the rising lobes. Assuming that the mass of cool gas, which is $\sim 60 \%$ of the total mass of gas remaining within $30 \mathrm{kpc}$ [4], was lifted out of the central cluster region by a continuous outflow or a series of bursts from the central AGN over the past 200-500 Myr (as it appears from the study of the cavity system, [117]), it would amount to outflows of a few hundred $M_{\odot} \mathrm{yr}^{-1}$. There would thus be a development of gas circulation that can significantly reduce the net inflow of cooling gas, as initially discussed by David et al. [4] and Nulsen et al. [110]. Therefore Gitti et al.'s [120] results show that the AGN feedback in Hydra A is acting not only by directly re-heating the gas, but also by removing a substantial amount of potential fuel for the supermassive black hole $(\mathrm{SMBH})$. This provides indications of mechanical AGN feedback acting through collimated, massive outflows generated by jets or cavity dragging (e.g., [217]).

The energy required to lift the cool gas gives a lower limit on the amount of AGN outburst energy deposited in the ICM. This value can be estimated by calculating the variation in gravitational potential energy during the lifting process. By assuming that the undisturbed ICM is approximately isothermal with sound speed $c_{s} \approx 1000 \mathrm{~km} \mathrm{~s}^{-1}$ and is in a 3 hydrostatic configuration with density profile $\rho(r)$, it is possible to calculate this quantity as [218]:

$$
\Delta E=\frac{M_{\mathrm{cool}} c_{s}^{2}}{\gamma} \ln \left(\frac{\rho_{i}}{\rho_{f}}\right),
$$

where $M_{\text {cool }}$ is the lifted mass, $\rho_{i}$ and $\rho_{f}$ are the initial and final densities of the surrounding ICM, and $\gamma=5 / 3$ is the ratio of specific heat capacities. From the density profile presented by David et al., Gitti et al. [120] estimated that the energy required to lift the cool gas is $\gtrsim 2.2 \times 10^{60} \mathrm{erg}$. This value is comparable to the work required to inflate all of the cavities against the surrounding pressure [117] and is $\sim 25 \%$ of the total energy of the large-scale shock [116]. Gitti et al. [120] also note that a good fraction of the energy required to lift the low entropy gas will be thermalized when the gas falls back inward. Given the large energy required, uplift provides a significant channel for the dissipation of outburst energy in Hydra A. There is a remarkable correlation between these low-entropy filaments and the metal-rich filaments in the iron-abundance maps measured by Simionescu et al. [219] and Kirkpatrick et al. [119], shown in Figure 6(b). The emerging picture is that Hydra A's powerful radio source is able to lift cool, metal-rich gas from the central region and distribute it throughout the X-ray atmosphere of the cluster. A similar effect of extended metal outflows in the direction of the radio lobes and X-ray cavities is observed in other systems (e.g., M87 [124, 220], A 262: [221], RBS 797 [111]). This is consistent with the results of the most current theoretical modeling of AGN feedback in massive cosmological systems, which predict the massive subrelativistic bipolar outflows and buoyant bubbles to produce a metal uplift along the jet axis (see Section 5.9).

5.9. Numerical Simulations. In the last decade the phenomenon of AGN feedback and its impact on the ICM has been the subject of many theoretical investigations (see $[92,93,163,174,202,203,222-230]$, to name a few). These works focused either on several aspects of feedback physics and microphysics or with the global, long-term evolution of the ICM. However, uncertainties are still large enough that the observations must guide researchers to select the relevant mechanisms at work in the feedback process.

The body of different observational investigations set a number of key constraints on process of AGN feedback. The results presented in this paper strongly suggest that AGN feedback manifests itself as massive subrelativistic bipolar outflows which heat the ICM through weak shocks and form $\mathrm{X}$-ray cavities, lift large masses of hot gas from the central region $\geq 100 \mathrm{kpc}$ and generate abundance asymmetries along the outflow direction. Processes such as AGN Compton heating or thermal conduction are unable to explain the collection of observations described above. Thus, although possibly relevant in some respect (e.g., [231]), they likely play a secondary role in local clusters and groups.

Recent 3D hydrosimulations of outflow feedback ([163, 203]; see, among others, also [36, 202, 217, 228, 232-235]) have quantitatively verified that collimated outflows reduce the gas cooling rate below the limits set by recent Chandra and XMM-Newton observations $([5,6]$ and references therein) for a timescale comparable with the cluster age. At the same time, this feedback mechanism generates ICM density and temperature profiles which reasonably agree with those of typical cool core clusters. This is not a trivial result $[93,174]$. The observable effects of the feedback from bipolar AGN outflows range from creation of X-ray cavities and large-scale shocks to inducing entropy and metal abundance anisotropies, due to the lifting of central gas, relatively cool and metal rich, along the direction of the jet (see also [236, 237]). These models, far from being complete and exhaustive 
(the origin of the outflows is essentially ad hoc), represent an interesting starting point for a more thorough understanding of the AGN feedback process.

\section{Concluding Remarks}

In order to fully understand the growth and evolution of galaxies and their central black holes, the history of star formation, and the formation of large-scale structures, it is crucial to understand first the processes of cooling, heating and the dynamical evolution of the intracluster gas. In particular, the feedback from the central black holes has turned out to be an essential ingredient that must be taken into account in any model of galaxy evolution. The main manifestation of the action of AGN feedback is in galaxy clusters and groups. Their study, which is currently a very active line of research in extragalactic astrophysics, has allowed us to make significant progresses in this field. However, many details of the AGN feedback mechanism are still unclear. It is not well understood, for instance, how the heating distributes in space and time in order to drastically reduce gas cooling, preserving at the same time the central cool core. An even more puzzling issue is the process of black hole accretion and feedback energy generation.

The last decade has represented a quantum leap in the quality of X-ray observations, thanks to the Chandra and $X M M-N e w t o n$ satellite telescopes. Recent results, discussed in this paper, have shaked up our understanding of the gas astrophysics in systems ranging from massive elliptical galaxies to rich galaxy clusters. They suggest that bipolar outflows emerging from the BCG core inflate large bubbles, heat the ICM and induce a circulation of gas and metals on scales of several $100 \mathrm{~s} \mathrm{kpc}$.

The current generation of X-ray observatories is still working well and can be expected to continue doing so for few more years. Unfortunately, the prospects for the future of X-ray astronomy are not clear at the moment, and Xray astronomers must rely on the good health on the existing, but aging, X-ray telescopes. As a result, the next few years represent a narrow time window to exploit the unique opportunity to observe deeply many additional objects, thus collecting crucial information on the cluster and group evolution. At the same time, current radio instrumentation is steadily improving both at the $\mathrm{MHz}$ and at the mm-wave ends of the spectrum and is about to make a significant step forward with the next generation of observatories, such as Low Frequency Array (LOFAR) and Atacama Large Millimeter/submillimeter Array (ALMA). A common effort, from both the observational and theoretical sides, will allow us to widen our knowledge on this fundamental problem which is central to the entire field of extragalactic astrophysics.

\section{Acknowledgments}

The authors especially thank Ewan O'Sullivan for sharing unpublished results and for providing useful comments. They thank Clif Kirkpatrick and Scott Randall for permission to print figures from their work. M. Gitti thanks Paul
Nulsen, Larry David, Jan Vrtilek, and Ewan O'Sullivan for many stimulating discussions on this exciting topic, and Stefano Ettori for carefully reading the original paper. M. Gitti acknowledges support by Grants ASI-INAF I/023/05/0 and I/088/06/0 and by Chandra grant GO0-11136X. B. R. McNamara acknowledges support from the Natural Sciences and Engineering Research Council of Canada.

\section{References}

[1] S. D. M. White and M. J. Rees, "Core condensation in heavy halos-a two-stage theory for galaxy formation and clustering," Monthly Notices of the Royal Astronomical Society, vol. 183, pp. 341-358, 1978.

[2] A. C. Fabian, "Cooling flows in clusters of galaxies," Annual Review of Astronomy and Astrophysics, vol. 32, no. 1, pp. 277318, 1994.

[3] J. R. Peterson, F. B. S. Paerels, J. S. Kaastra et al., "X-ray imaging-spectroscopy of Abell 1835," Astronomy and Astrophysics, vol. 365, no. 1, pp. L104-L109, 2001.

[4] L. P. David, P. E. J. Nulsen, B. R. Mcnamara et al., "A high-resolution study of the Hydra A cluster with Chandra: comparison of the core mass distribution with theoretical predictions and evidence for feedback in the cooling flow," Astrophysical Journal, vol. 557, no. 2, pp. 546-559, 2001.

[5] J. R. Peterson and A. C. Fabian, "X-ray spectroscopy of cooling clusters," Physics Reports, vol. 427, no. 1, pp. 1-39, 2006.

[6] B. R. McNamara and P. E. J. Nulsen, "Heating hot atmospheres with active galactic nuclei," Annual Review of Astronomy and Astrophysics, vol. 45, pp. 117-175, 2007.

[7] J. O. Burns, "The radio properties of $\mathrm{cD}$ galaxies in abell clusters. I. An X-ray-selected sample,” Astronomical Journal, vol. 99, no. 1, pp. 14-30, 1990.

[8] L. Bîrzan, B. R. McNamara, P. E. J. Nulsen, C. L. Carilli, and M. W. Wise, "Radiative efficiency and content of extragalactic radio sources: toward a universal scaling relation between jet power and radio power," Astrophysical Journal, vol. 686, no. 2, pp. 859-880, 2008.

[9] S. Giacintucci, E. O'Sullivan, J. Vrtilek et al., "A combined low-radio frequency/X-ray study of galaxy groups. I. Giant metrewave radio telescope observations at $235 \mathrm{MHz}$ and $610 \mathrm{MHz}$," Astrophysical Journal Letters, vol. 732, article 95, no. 2, 2011.

[10] J. Magorrian, S. Tremaine, D. Richstone et al., "The demography of massive dark objects in galaxy centers," Astronomical Journal, vol. 115, no. 6, pp. 2285-2305, 1998.

[11] A. J. Benson, R. G. Bower, C. S. Frenk, C. G. Lacey, C. M. Baugh, and S. Cole, "What shapes the luminosity function of galaxies?" Astrophysical Journal, vol. 599, no. 1, pp. 38-49, 2003.

[12] D. J. Croton, V. Springel, S. D. M. White et al., "The many lives of active galactic nuclei: cooling flows, black holes and the luminosities and colours of galaxies," Monthly Notices of the Royal Astronomical Society, vol. 365, no. 1, pp. 11-28, 2006.

[13] M. L. Balogh, A. Babul, G. M. Voit et al., "An analytic investigation of the scatter in the integrated X-ray properties of galaxy groups and clusters," Monthly Notices of the Royal Astronomical Society, vol. 366, no. 2, pp. 624-634, 2006.

[14] A. Cattaneo, S. M. Faber, J. Binney et al., "The role of black holes in galaxy formation and evolution," Nature, vol. 460, no. 7252, pp. 213-219, 2009. 
[15] C. L. Sarazin, X-Ray Emission from Clusters of Galaxies, 1988.

[16] R. F. Mushotzky, "Clusters of galaxies: an X-ray perspective," http://arxiv.org/abs/astro-ph/0311105.

[17] M. Arnaud, "X-ray observations of clusters of galaxies," in Background Microwave Radiation and Intracluster Cosmology, F. Melchiorri and Y. Rephaeli, Eds., p. 77, 2005.

[18] G. M. Voit, "Tracing cosmic evolution with clusters of galaxies," Reviews of Modern Physics, vol. 77, no. 1, pp. 207-258, 2005.

[19] M. Markevitch and A. Vikhlinin, "Shocks and cold fronts in galaxy clusters," Physics Reports, vol. 443, no. 1, pp. 1-53, 2007.

[20] P. Rosati, S. Borgani, and C. Norman, "The evolution of Xray clusters of galaxies," Annual Review of Astronomy and Astrophysics, vol. 40, pp. 539-577, 2002.

[21] W. H. Press and P. Schechter, "Formation of galaxies and clusters of galaxies by self-similar gravitational condensation," Astrophysical Journal, vol. 187, pp. 425-438, 1974.

[22] S. Borgani, "Cosmology with clusters of galaxies," http://arxiv .org/abs/astro-ph/0605575.

[23] N. Kaiser, "Evolution and clustering of rich clusters," Monthly Notices of the Royal Astronomical Society, vol. 222, pp. 323345, 1986.

[24] G. L. Bryan and M. L. Norman, "Statistical properties of x-ray clusters: analytic and numerical comparisons," Astrophysical Journal, vol. 495, no. 1, pp. 80-99, 1998.

[25] M. Markevitch, "The LX-T relation and temperature function for nearby clusters revisited," Astrophysical Journal, vol. 504, no. 1, pp. 27-34, 1998.

[26] M. Arnaud and A. E. Evrard, "The LX-T relation and intracluster gas fractions of X-ray clusters," Monthly Notices of the Royal Astronomical Society, vol. 305, no. 3, pp. 631-640, 1999.

[27] S. Ettori, P. Tozzi, S. Borgani, and P. Rosati, "Scaling laws in X-ray galaxy clusters at redshift between 0.4 and 1.3," Astronomy and Astrophysics, vol. 417, no. 1, pp. 13-27, 2004.

[28] B. J. Maughan, L. R. Jones, H. Ebeling, and C. Scharf, "The evolution of the cluster X-ray scaling relations in the Wide Angle ROSAT Pointed Survey sample at $0.6<\mathrm{z}<1.0$," Monthly Notices of the Royal Astronomical Society, vol. 365, no. 2, pp. 509-529, 2006.

[29] N. Ota, T. Kitayama, K. Masai, and K. Mitsuda, "LX-T relation and related properties of galaxy clusters," Astrophysical Journal, vol. 640, no. 2, pp. 673-690, 2006.

[30] A. Mantz, S. W. Allen, D. Rapetti, and H. Ebeling, "The observed growth of massive galaxy clusters-I. Statistical methods and cosmological constraints," Monthly Notices of the Royal Astronomical Society, vol. 406, no. 3, pp. 1759-1772, 2010.

[31] N. Kaiser, "Evolution of clusters of galaxies," Astrophysical Journal, vol. 383, no. 1, pp. 104-111, 1991.

[32] A. E. Evrard and J. P. Henry, "Expectations for X-ray cluster observations by the ROSAT satellite," Astrophysical Journal, vol. 383, no. 1, pp. 95-103, 1991.

[33] S. Cole, "Modeling galaxy formation in evolving dark matter halos," Astrophysical Journal, vol. 367, no. 1, pp. 45-53, 1991.

[34] S. D. M. White and C. S. Frenk, "Galaxy formation through hierarchical clustering," Astrophysical Journal, vol. 379, no. 1, pp. 52-79, 1991.

[35] M. L. Balogh, F. R. Pearce, R. G. Bower, and S. T. Kay, "Revisiting the cosmic cooling crisis," Monthly Notices of the Royal Astronomical Society, vol. 326, no. 4, pp. 1228-1234, 2001.
[36] D. Sijacki and V. Springel, "Hydrodynamical simulations of cluster formation with central AGN heating," Monthly Notices of the Royal Astronomical Society, vol. 366, no. 2, pp. 397-416, 2006.

[37] C. A. Metzler and A. E. Evrard, "A simulation of the intracluster medium with feedback from cluster galaxies," Astrophysical Journal, vol. 437, no. 2, pp. 564-583, 1994.

[38] S. Borgani, F. Governato, J. Wadsley et al., "The effect of nongravitational gas heating in groups and clusters of galaxies," Monthly Notices of the Royal Astronomical Society, vol. 336, no. 2, pp. 409-424, 2002.

[39] A. Finoguenov, M. Arnaud, and L. P. David, "Temperature and heavy-element abundance profiles of cool clusters of galaxies from ASCA," Astrophysical Journal, vol. 555, no. 1, pp. 191-204, 2001.

[40] A. Pipino, F. Matteucci, S. Borgani, and A. Biviano, "SNe heating and the chemical evolution of the intra-cluster medium," New Astronomy, vol. 7, no. 5, pp. 227-247, 2002.

[41] L. Tornatore, S. Borgani, V. Springel, F. Matteucci, N. Menci, and G. Murante, "Cooling and heating the intracluster medium in hydrodynamical simulations," Monthly Notices of the Royal Astronomical Society, vol. 342, no. 4, pp. 1025-1040, 2003.

[42] G. M. Voit, G. L. Bryan, M. L. Balogh, and R. G. Bower, "Modified entropy models for the intracluster medium," Astrophysical Journal, vol. 576, no. 2, pp. 601-624, 2002.

[43] K. K. S. Wu, A. C. Fabian, and P. E. J. Nulsen, "The soft Xray background: evidence for widespread disruption of the gas haloes of galaxy groups," Monthly Notices of the Royal Astronomical Society, vol. 324, no. 1, pp. 95-107, 2001.

[44] E. Scannapieco and S. P. Oh, "Quasar feedback: the missing link in structure formation," Astrophysical Journal, vol. 608, no. 1, pp. 62-79, 2004.

[45] M. Donahue, D. J. Horner, K. W. Cavagnolo, and G. M. Voit, "Entropy profiles in the cores of cooling flow clusters of galaxies," Astrophysical Journal, vol. 643, no. 2, pp. 730-750, 2006.

[46] G. M. Voit and M. Donahue, "An observationally motivated framework for agn heating of cluster cores," Astrophysical Journal, vol. 634, no. 2, pp. 955-963, 2005.

[47] L. Ferrarese and D. Merritt, "A fundamental relation between supermassive black holes and their host galaxies," Astrophysical Journal, vol. 539, no. 1, pp. L9-L12, 2000.

[48] K. Gebhardt, R. Bender, G. Bower et al., "A relationship between nuclear black hole mass and galaxy velocity dispersion," Astrophysical Journal, vol. 539, no. 1, pp. L13-L16, 2000.

[49] S. Tremaine, K. Gebhardt, R. Bender et al., "The slope of the black hole mass versus velocity dispersion correlation," Astrophysical Journal, vol. 574, no. 2, pp. 740-753, 2002.

[50] J. E. Felten, R. J. Gould, W. A. Stein, and N. J. Woolf, "Xrays from the coma cluster of galaxies," Astrophysical Journal Letters, vol. 146, pp. 955-958, 1966.

[51] L. J. Spitzer, Physics of Fully Ionized Gases, 1956.

[52] L. J. Spitzer, Physical Processes in the Interstellar Medium, 1978.

[53] R. K. Smith and J. P. Hughes, "Ionization equilibrium timescales in collisional plasmas," Astrophysics Journal, vol. 718, pp. 583-585, 2010.

[54] G. B. Rybicki and A. P. Lightman, Radiative Processes in Astrophysics, Wiley-VCH, 1979.

[55] W. J. Karzas and R. Latter, "Electron radiative transitions in a coulomb field," Astrophysical Journal Letters, vol. 6, p. 167, 1961. 
[56] E. Kellogg, J. R. Baldwin, and D. Koch, "Studies of cluster Xray sources, energy spectra for the Perseus, Virgo, and Coma clusters," Astrophysical Journal Letters, vol. 199, pp. 299-306, 1975.

[57] J. C. Raymond and B. W. Smith, "Soft X-ray spectrum of a hot plasma," Astrophysical Journal Supplement Series, vol. 35, pp. 419-439, 1977.

[58] R. Mewe and E. H. B. M. Gronenschild, "Calculated Xradiation from optically thin plasmas. IV-atomic data and rate coefficients for spectra in the range 1-270 A," Astronomy and Astrophysics Supplement Series, vol. 45, pp. 11-52, 1981.

[59] C. L. Sarazin and J. N. Bahcall, "X-ray line emission for clusters of galaxies. II-numerical models," Astrophysical Journal Supplement Series, vol. 34, pp. 451-467, 1977.

[60] J. N. Bahcall and C. L. Sarazin, "Parameters and predictions for the X-ray emitting gas of Coma, Perseus, and Virgo," Astrophysical Journal, vol. 213, pp. L99-L103, 1977.

[61] J. N. Bahcall and C. L. Sarazin, "X-ray line spectroscopy for clusters of galaxies. I," Astrophysical Journal, vol. 219, pp. 781-794, 1978.

[62] C. F. McKee and L. L. Cowie, "The evaporation of spherical clouds in a hot gas. II-effects of radiation," Astrophysical Journal, vol. 215, pp. 213-225, 1977.

[63] R. S. Sutherland and M. A. Dopita, "Cooling functions for low-density astrophysical plasmas," Astrophysical Journal, Supplement Series, vol. 88, no. 1, pp. 253-327, 1993.

[64] L. M. Voigt and A. C. Fabian, "Galaxy cluster mass profiles," Monthly Notices of the Royal Astronomical Society, vol. 368, no. 2, pp. 518-533, 2006.

[65] M. Gitti, R. Piffaretti, and S. Schindler, "Mass distribution in the most X-ray-luminous galaxy cluster RX J1347.5-1145 studied with XMM-Newton," Astronomy and Astrophysics, vol. 472, no. 2, pp. 383-394, 2007.

[66] F. Gastaldello, D. A. Buote, P. J. Humphrey et al., "Probing the dark matter and gas fraction in relaxed galaxy groups with X-ray observations from Chandra and XMM-Newton," Astrophysical Journal, vol. 669, no. 1, pp. 158-183, 2007.

[67] S. Ettori, F. Gastaldello, A. Leccardi et al., "Mass profiles and c-MDM relation in X-ray luminous galaxy clusters," Astronomy and Astrophysics, vol. 524, no. 3, article 68, 2010.

[68] E. Rasia, S. Ettori, L. Moscardini et al., "Systematics in the X-ray cluster mass estimators," Monthly Notices of the Royal Astronomical Society, vol. 369, no. 4, pp. 2013-2024, 2006.

[69] D. Nagai, A. V. Kravtsov, and A. Vikhlinin, "Effects of galaxy formation on thermodynamics of the intracluster medium," Astrophysical Journal, vol. 668, no. 1, pp. 1-14, 2007.

[70] R. Piffaretti and R. Valdarnini, "Total mass biases in X-ray galaxy clusters," Astronomy and Astrophysics, vol. 491, no. 1, pp. 71-87, 2008.

[71] A. Cavaliere and R. Fusco-Femiano, "X-rays from hot plasma in clusters of galaxies," Astronomy and Astrophysics, vol. 49, pp. 137-144, 1976.

[72] M. Arnaud, "The beta of the intracluster medium," Astronomy and Astrophysics, vol. 500, no. 1, pp. 103-104, Commentary on: A. Cavaliere and R. Fusco-Femiano, Astronomy and Astrophysics, vol. 49, pp. 137, 1976, 2009.

[73] I. King, "The structure of star clusters. I. An empirical density law," Astronomical Journal, vol. 67, p. 471, 1962.

[74] S. Ettori and F. Brighenti, "On the evolution of cooling cores in X-ray galaxy clusters," Monthly Notices of the Royal Astronomical Society, vol. 387, no. 2, pp. 631-638, 2008.
[75] Y. Chen, T. H. Reiprich, H. Böhringer, Y. Ikebe, and Y. Y. Zhang, "Statistics of X-ray observables for the cooling-core and non-cooling core galaxy clusters," Astronomy and Astrophysics, vol. 466, no. 3, pp. 805-812, 2007.

[76] S. De Grandi and S. Molendi, "Metallicity gradients in X-ray clusters of galaxies," Astrophysical Journal, vol. 551, no. 1, pp. 153-159, 2001.

[77] S. de Grandi and S. Molendi, "Metal abundances in the coolcores of galaxy clusters," Astronomy and Astrophysics, vol. 508, pp. 565-574, 2009.

[78] S. W. Allen, R. W. Schmidt, and A. C. Fabian, "The Xray virial relations for relaxed lensing clusters observed with Chandra," Monthly Notices of the Royal Astronomical Society, vol. 328, no. 3, pp. L37-L41, 2001.

[79] A. Vikhlinin, M. Markevitch, S. S. Murray, C. Jones, W. Forman, and L. Van Speybroeck, "Chandra temperature profiles for a sample of nearby relaxed galaxy clusters," Astrophysical Journal, vol. 628, no. 2, pp. 655-672, 2005.

[80] D. S. Hudson, R. Mittal, T. H. Reiprich, P. E. J. Nulsen, H. Andernach, and C. L. Sarazin, "What is a cool-core cluster? a detailed analysis of the cores of the X-ray flux-limited HIFLUGCS cluster sample," Astronomy and Astrophysics, vol. 513, article A37, p. 37, 2010.

[81] R. M. Johnstone, S. W. Allen, A. C. Fabian, and J. S. Sanders, "Chandra observations of Abell 2199," Monthly Notices of the Royal Astronomical Society, vol. 336, no. 1, pp. 299-308, 2002.

[82] H. Böhringer and N. Werner, "X-ray spectroscopy of galaxy clusters: studying astrophysical processes in the largest celestial laboratories," Astronomy and Astrophysics Review, vol. 18, no. 1-2, pp. 127-196, 2010.

[83] A. C. Fabian, R. F. Mushotzky, P. E. J. Nulsen, and J. R. Peterson, "On the soft X-ray spectrum of cooling flows," Monthly Notices of the Royal Astronomical Society, vol. 321, no. 1, pp. L20-L24, 2001.

[84] R. G. Morris and A. C. Fabian, "Some effects of small-scale metallicity variations in cooling flows," Monthly Notices of the Royal Astronomical Society, vol. 338, no. 4, pp. 824-836, 2003.

[85] A. C. Fabian, S. W. Allen, C. S. Crawford et al., "The missing soft X-ray luminosity in cluster cooling flows," Monthly Notices of the Royal Astronomical Society, vol. 332, no. 3, pp. L50-L54, 2002.

[86] W. G. Mathews and F. Brighenti, "Rapid cooling of dusty gas in elliptical galaxies," Astrophysical Journal, vol. 590, no. 1, pp. L5-L8, 2003.

[87] R. Rosner and W. H. Tucker, "On magnetic fields, heating and thermal conduction in halos, and the suppression of cooling flows," Astrophysical Journal, vol. 338, pp. 761-769, 1989.

[88] G. Tabor and J. Binney, "Elliptical galaxy cooling flows without mass drop-out," Monthly Notices of the Royal Astronomical Society, vol. 263, p. 323, 1993.

[89] E. Churazov, M. Brüggen, C. R. Kaiser, H. Böhringer, and W. Forman, "Evolution of buoyant bubbles in M87," Astrophysical Journal, vol. 554, no. 1, pp. 261-273, 2001.

[90] M. Brüggen and C. R. Kaiser, "Buoyant radio plasma in clusters of galaxies," Monthly Notices of the Royal Astronomical Society, vol. 325, no. 2, pp. 676-684, 2001.

[91] C. R. Kaiser and J. Binney, "Simple models of cooling flows," Monthly Notices of the Royal Astronomical Society, vol. 338, no. 4, pp. 837-845, 2003.

[92] M. Ruszkowski and M. C. Begelman, "Heating, conduction, and minimum temperatures in cooling flows," Astrophysical Journal, vol. 581, no. 1, pp. 223-228, 2002. 
[93] F. Brighenti and W. G. Mathews, "Feedback heating in cluster and galactic cooling flows," Astrophysical Journal, vol. 587, no. 2, pp. 580-588, 2003.

[94] W. H. Tucker and R. Rosner, "Thermal conduction and heating by nonthermal electrons in the X-ray halo of M87," Astrophysical Journal, vol. 267, pp. 547-550, 1983.

[95] L. M. Voigt, R. W. Schmidt, A. C. Fabian, S. W. Allen, and R. M. Johnstone, "Conduction and cooling flows," Monthly Notices of the Royal Astronomical Society, vol. 335, no. 1, pp. L7-L11, 2002.

[96] A. C. Fabian, L. M. Voigt, and R. G. Morris, "On conduction, cooling flows and galaxy formation," Monthly Notices of the Royal Astronomical Society, vol. 335, no. 3, pp. L71-L74, 2002.

[97] N. L. Zakamska and R. Narayan, "Models of galaxy clusters with thermal conduction," Astrophysical Journal, vol. 582, no. 1, pp. 162-169, 2003.

[98] M. Markevitch, A. Vikhlinin, and P. Mazzotta, "Nonhydrostatic gas in the core of the relaxed galaxy cluster A1795," Astrophysical Journal, vol. 562, no. 2, pp. L153-L156, 2001.

[99] A. C. Fabian, "A gravitational contribution to the cooling flow problem," Monthly Notices of the Royal Astronomical Society, vol. 344, no. 2, pp. L27-L30, 2003.

[100] W. Domainko, M. Gitti, S. Schindler, and W. Kapferer, "Feedback from intra-cluster supernovae on the ICM in cooling flow galaxy clusters," Astronomy and Astrophysics, vol. 425, no. 2, pp. L21-L24, 2004.

[101] P. N. Best, A. von der Linden, G. Kauffmann, T. M. Heckman, and C. R. Kaiser, "On the prevalence of radio-loud active galactic nuclei in brightest cluster galaxies: implications for AGN heating of cooling flows," Monthly Notices of the Royal Astronomical Society, vol. 379, no. 3, pp. 894-908, 2007.

[102] R. Mittal, D. S. Hudson, T. H. Reiprich, and T. Clarke, "AGN heating and ICM cooling in the HIFLUGCS sample of galaxy clusters," Astronomy and Astrophysics, vol. 501, no. 3, pp. 835850, 2009.

[103] E. Churazov, S. Sazonov, R. Sunyaev, W. Forman, C. Jones, and H. Böhringer, "Supermassive black holes in elliptical galaxies: switching from very bright to very dim," Monthly Notices of the Royal Astronomical Society, vol. 363, no. 1, pp. L91-L95, 2005.

[104] H. Boehringer, W. Voges, A. C. Fabian, A. C. Edge, and D. M. Neumann, "A ROSAT HRI study of the interaction of the X-ray-emitting gas and radio lobes of NGC 1275," Monthly Notices of the Royal Astronomical Society, vol. 264, pp. L25L28, 1993.

[105] C. L. Carilli, R. A. Perley, and D. E. Harris, "Observations of interaction between cluster gas and the radio lobes of cygnusA," Monthly Notices of the Royal Astronomical Society, vol. 270, pp. 173-177, 1994.

[106] A. C. Fabian, J. S. Sanders, S. Ettori et al., "Chandra imaging of the complex X-ray core of the Perseus cluster," Monthly Notices of the Royal Astronomical Society, vol. 318, no. 4, pp. L65-L68, 2000.

[107] B. R. McNamara, M. Wise, P. E. J. Nulsen et al., "Chandra $\mathrm{X}$-ray observations of the hydra a cluster: an interaction between the radio source and the X-ray-emitting gas," Astrophysical Journal, vol. 534, no. 2, pp. L135-L138, 2000.

[108] E. L. Blanton, C. L. Sarazin, B. R. Mcnamara, and M. W. Wise, "Chandra observation of the radio source/Xray gas interaction in the cooling flow cluster abell 2052," Astrophysical Journal, vol. 558, no. 1, pp. L15-L18, 2001.

[109] E. L. Blanton, C. L. Sarazin, and B. R. McNamara, "Chandra observation of the cooling flow cluster abell 2052," Astrophysical Journal, vol. 585, no. 1, pp. 227-243, 2003.
[110] P. E. J. Nulsen, L. P. David, B. R. McNamara, C. Jones, W. R. Forman, and M. Wise, "Interaction of radio lobes with the hot intracluster medium: driving convective outflow in Hydra A," Astrophysical Journal, vol. 568, no. 1, pp. 163-173, 2002.

[111] A. Doria, M. Gitti, S. Ettori, F. Brighenti, P. E. J. Nulsen, and B. R. McNamara, "A chandra-VLA investigation of the Xray cavity system and radio mini-halo in the galaxy cluster RBS 797," submitted to Astrophysical Journal Letters.

[112] R. J. H. Dunn and A. C. Fabian, "Investigating AGN heating in a sample of nearby clusters," Monthly Notices of the Royal Astronomical Society, vol. 373, no. 3, pp. 959-971, 2006.

[113] L. Bîrzan, D. A. Rafferty, B. R. McNamara, P. E. J. Nulsen, and M. W. Wise, "The detectability of AGN cavities in coolingflow clusters," in Proceedings of the American Institute of Physics Conference Proceedings, S. Heinz and E. Wilcots, Eds., vol. 1201, pp. 301-304, 2009.

[114] E. Churazov, W. Forman, C. Jones, and H. Böhringer, "Asymmetric, arc minute scale structures around NGC 1275," Astronomy and Astrophysics, vol. 356, no. 3, pp. 788-794, 2000.

[115] A. C. Fabian, J. S. Sanders, G. B. Taylor et al., "A very deep Chandra observation of the Perseus cluster: shocks, ripples and conduction," Monthly Notices of the Royal Astronomical Society, vol. 366, no. 2, pp. 417-428, 2006.

[116] P. E. J. Nulsen, B. R. Mcnamara, M. W. Wise, and L. P. David, "The cluster-scale AGN outburst in Hydra A," Astrophysical Journal, vol. 628, no. 2, pp. 629-636, 2005.

[117] M. W. Wise, B. R. McNamara, P. E. J. Nulsen, J. C. Houck, and L. P. David, "X-ray supercavities in the hydra a cluster and the outburst history of the central galaxy's active nucleus," Astrophysical Journal, vol. 659, no. 2, pp. 1153-1158, 2007.

[118] A. Simionescu, E. Roediger, P. E. J. Nulsen et al., "The largescale shock in the cluster of galaxies Hydra A," Astronomy and Astrophysics, vol. 495, no. 3, pp. 721-732, 2009.

[119] C. C. Kirkpatrick, M. Gitti, K. W. Cavagnolo et al., "Direct evidence for outflow of metal-enriched gas along the radio jets of hydra A," Astrophysical Journal Letters, vol. 707, no. 1, part 2, pp. L69-L72, 2009.

[120] M. Gitti, P. E. J. Nulsen, L. P. David, B. R. McNamara, and M. W. Wise, "A Chandra study of the large-scale shock and cool filaments in Hydra A: evidence for substantial gas dredgeup by the central outburst," Astrophysical Journal Letters, vol. 732, article 13, no. 1, 2011.

[121] H. Bohringer, P. E. J. Nulsen, R. Braun, and A. C. Fabian, "The interaction of the radio halo of M87 with the cooling intracluster medium of the Virgo cluster," Monthly Notices of the Royal Astronomical Society, vol. 274, pp. L67-L71, 1995.

[122] S. Molendi, "On the temperature structure of M87," Astrophysical Journal, vol. 580, no. 2, pp. 815-823, 2002.

[123] W. Forman, C. Jones, E. Churazov et al., "Filaments, bubbles, and weak shocks in the gaseous atmosphere of M87," Astrophysical Journal, vol. 665, no. 2, pp. 1057-1066, 2007.

[124] A. Simionescu, N. Werner, A. Finoguenov, H. Böhringer, and M. Brüggen, "Metal-rich multi-phase gas in M 87 AGNdriven metal transport, magnetic-field supported multitemperature gas, and constraints on non-thermal emission observed with XMM-Newton," Astronomy and Astrophysics, vol. 482, no. 1, pp. 97-112, 2008.

[125] E. T. Million, N. Werner, A. Simionesc et al., "Feedback under the microscope-I. Thermodynamic structure and AGN-driven shocks in M87," Monthly Notices of the Royal Astronomical Society, vol. 407, no. 4, pp. 2046-2062, 2010.

[126] E. L. Blanton, C. L. Sarazin, B. R. Mcnamara, and T. E. Clarke, "Chandra observation of the central region of the cooling 
flow cluster A262: a radio source that is a shadow of its former self?" Astrophysical Journal, vol. 612, no. 2, pp. 817-824, 2004.

[127] E. L. Blanton, S. W. Randall, E. M. Douglass, C. L. Sarazin, T. E. Clarke, and B. R. McNamara, "Shocks and bubbles in a deep chandra observation of the cooling flow cluster Abell 2052," Astrophysical Journal, vol. 697, no. 2, pp. L95-L98, 2009.

[128] E. L. Blanton, S. W. Randall, T. E. Clarke et al., "A very deep Chandra observation of abell 2052: bubbles, shocks, and sloshing," The Astrophysical Journal, vol. 737, no. 2, article 99, 2011.

[129] S. Schindler, A. Castillo-Morales, E. De Filippis, A. Schwope, and J. Wambsganss, "Discovery of depressions in the X-ray emission of the distant galaxy cluster RBS797 in a CHANDRA observation," Astronomy and Astrophysics, vol. 376, no. 3, pp. L27-L30, 2001.

[130] M. Gitti, L. Feretti, and S. Schindler, "Multifrequency VLA radio observations of the X-ray cavity cluster of galaxies RBS797: evidence of differently oriented jets," Astronomy and Astrophysics, vol. 448, no. 3, pp. 853-860, 2006.

[131] K. W. Cavagnolo, B. R. McNamara, M. W. Wise et al., "A powerful AGN outburst in RBS 797," Astrophysical Journal Letters, vol. 732, article 71, no. 2, 2011.

[132] Y. Fujita, C. L. Sarazin, J. C. Kempner et al., "Chandra observations of the disruption of the cool core in A133," Astrophysical Journal, vol. 575, no. 2, pp. 764-778, 2002.

[133] S. W. Randall, T. E. Clarke, P. E. J. Nulsen et al., "Radio and deep Chandra observations of the disturbed cool core cluster Abell 133," Astrophysical Journal, vol. 722, no. 1, pp. 825-846, 2010.

[134] T. E. Clarke, E. L. Blanton, C. L. Sarazin et al., “Tracing multiple generations of active galactic nucleus feedback in the core of abell 262," Astrophysical Journal, vol. 697, no. 2, pp. 1481-1492, 2009.

[135] B. R. McNamara, P. E. J. Nulsen, M. W. Wise et al., “The heating of gas in a galaxy cluster by X-ray cavities and large-scale shock fronts," Nature, vol. 433, no. 7021, pp. 45-47, 2005.

[136] M. Gitti, B. R. McNamara, P. E. J. Nulsen, and M. W. Wise, "Cosmological effects of powerful AGN outbursts in galaxy clusters: insights from an XMM-Newton observation of MS 0735+7421," Astrophysical Journal, vol. 660, no. 2, pp. 11181136, 2007.

[137] L. Bîrzan, D. A. Rafferty, B. R. McNamara, M. W. Wise, and P. E. J. Nulsen, "A systematic study of radio-induced Xray cavities in clusters, groups, and galaxies," Astrophysical Journal, vol. 607, no. 2, pp. 800-809, 2004.

[138] R. J. H. Dunn, A. C. Fabian, and G. B. Taylor, "Radio bubbles in clusters of galaxies," Monthly Notices of the Royal Astronomical Society, vol. 364, no. 4, pp. 1343-1353, 2005.

[139] D. A. Rafferty, B. R. Mcnamara, P. E. J. Nulsen, and M. W. Wise, "The feedback-regulated growth of black holes and bulges through gas accretion and starbursts in cluster central dominant galaxies," Astrophysical Journal, vol. 652, no. 1, pp. 216-231, 2006.

[140] S. Diehl, H. Li, C. L. Fryer, and D. Rafferty, "Constraining the nature of X-ray cavities in clusters and galaxies," Astrophysical Journal, vol. 687, no. 1, pp. 173-192, 2008.

[141] D. E. Harris, A. Finoguenov, A. H. Bridle, M. J. Hardcastle, and R. A. Laing, "X-ray detection of the inner jet in the radio galaxy M84," Astrophysical Journal, vol. 580, no. 1, pp. 110$113,2002$.

[142] A. Finoguenov, M. Ruszkowski, C. Jones, M. Brüggen, A. Vikhlinin, and E. Mandel, "In-depth Chandra study of the
AGN feedback in virgo elliptical galaxy M84," Astrophysical Journal, vol. 686, no. 2, pp. 911-917, 2008.

[143] C. Jones, W. Forman, A. Vikhlinin et al., "Chandra observations of NGC 4636-an elliptical galaxy in turmoil," Astrophysical Journal, vol. 567, no. 2, pp. L115-L118, 2002.

[144] E. O'Sullivan, J. M. Vrtilek, and J. C. Kempner, “Active galactic nucleus feedback and gas mixing in the core of NGC 4636," Astrophysical Journal, vol. 624, no. 2, pp. L77-L80, 2005.

[145] A. Baldi, W. Forman, C. Jones et al., "The unusual X-ray morphology of NGC 4636 revealed by deep chandra observations: cavities and shocks created by past active galactic nucleus outbursts," Astrophysical Journal, vol. 707, no. 2, pp. 1034-1043, 2009.

[146] D. A. Buote, A. D. Lewis, F. Brighenti, and W. G. Mathews, "XMM-Newton and Chandra observations of the galaxy group NGC 5044. I. Evidence for limited multiphase hot gas," Astrophysical Journal, vol. 594, no. 2, pp. 741-757, 2003.

[147] F. Gastaldello, D. A. Buote, P. Temi, F. Brighenti, W. G. Mathews, and S. Ettori, "X-ray cavities, filaments, and cold fronts in the core of the galaxy group NGC 5044," Astrophysical Journal Letters, vol. 693, pp. 43-55, 2009.

[148] L. P. David, C. Jones, W. Forman et al., "Isotropic active galactic nucleus heating with small radio-quiet bubbles in the ngc 5044 group," Astrophysical Journal, vol. 705, no. 1, pp. 624-638, 2009.

[149] L. P. David, E. O’Sullivan, C. Jones et al., “Active-galacticnucleus-driven weather and multiphase gas in the core of the NGC 5044 galaxy group," Astrophysical Journal Letters, vol. 728, article 162, no. 2, 2011.

[150] J. M. Vrtilek, L. Grego, L. P. David et al., "A Sharper Picture of X-Ray Bright Galaxy Groups: Chandra Imaging and Spectroscopy of HCG 62 and NGC 741," in Proceedings of the American Physical Society Meeting, Aprril 2002.

[151] U. Morita, Y. Ishisaki, N. Y. Yamasaki et al., "Chandra and XMM-Newton observations of a group of galaxies, HCG 62," Publications of the Astronomical Society of Japan, vol. 58, no. 4, pp. 719-742, 2006.

[152] J. Gu, H. Xu, L. Gu et al., "A high-abundance arc in the compact group of galaxies HCG 62: an AGN- or merger-induced metal outflow?" Astrophysical Journal, vol. 659, no. 1, pp. 275-282, 2007.

[153] M. Gitti, E. O’Sullivan, S. Giacintucci et al., "Cavities and shocks in the galaxy group HCG 62 as revealed by Chandra, XMM-Newton, and giant metrewave radio telescope data," Astrophysical Journal Letters, vol. 714, no. 1, pp. 758-771, 2010.

[154] M. E. Machacek, D. Jerius, R. P. Kraft et al., “Deep Chandra observations of edges and bubbles in the NGC 5846 galaxy group," The Astrophysical Journal, vol. 743, no. 1, article 15, 2011.

[155] V. R. Eke, C. S. Frenk, C. M. Baugh et al., "Galaxy groups in the two-degree field galaxy redshift survey: the luminous content of the groups," Monthly Notices of the Royal Astronomical Society, vol. 355, no. 3, pp. 769-784, 2004.

[156] S. Giodini, V. Smolić, A. Finoguenov et al., "Radio galaxy feedback in x-ray-selected groups from cosmos: the effect on the intracluster medium," Astrophysical Journal, vol. 714, no. 1, pp. 218-228, 2010.

[157] S. W. Randall, W. R. Forman, S. Giacintucci et al., "Shocks and cavities from multiple outbursts in the galaxy group NGC 5813: a window to active galactic nucleus feedback," Astrophysical Journal Letters, vol. 726, article 86, no. 2, 2011. 
[158] E. O'Sullivan, S. Giacintucci, L. P. David, J. M. Vrtilek, and S. Raychaudhury, "A deep Chandra observation of the poor cluster AWM 4-I. Properties of the central radio galaxy and its effects on the intracluster medium," Monthly Notices of the Royal Astronomical Society, vol. 407, no. 1, pp. 321-338, 2010.

[159] E. O'Sullivan, S. Giacintucci, L. P. David, J. M. Vrtilek, and S. Raychaudhury, "A deep Chandra observation of the poor cluster AWM 4-II. The role of the radio jets in enriching the intracluster medium," Monthly Notices of the Royal Astronomical Society, vol. 411, no. 3, pp. 1833-1842, 2011.

[160] R. Johnson, T. J. Ponman, and A. Finoguenov, "A statistical analysis of the Two-Dimensional XMM-Newton Group Survey: the impact of feedback on group properties," Monthly Notices of the Royal Astronomical Society, vol. 395, no. 3, pp. 1287-1308, 2009.

[161] M. Sun, "Every BCG with a strong radio AGN has an $\mathrm{x}$ ray cool core: is the cool core-noncool core dichotomy too simple?" Astrophysical Journal, vol. 704, no. 2, pp. 1586-1604, 2009.

[162] R. Dong, J. Rasmussen, and J. S. Mulchaey, "A systematic search for X-ray cavities in the hot gas of galaxy groups," Astrophysical Journal, vol. 712, no. 2, pp. 883-900, 2010.

[163] M. Gaspari, F. Brighenti, A. D’Ercole, and C. Melioli, “AGN feedback in galaxy groups: the delicate touch of self-regulated outflows," Monthly Notices of the Royal Astronomical Society, vol. 415, no. 2, pp. 1549-1568, 2011.

[164] W. M. Lane, T. E. Clarke, G. B. Taylor, R. A. Perley, and N. E. Kassim, "Hydra a at low radio frequencies," Astronomical Journal, vol. 127, no. 1, pp. 48-52, 2004.

[165] E. O'Sullivan, S. Giacintucci, L. P. David et al., "Heating the hot atmospheres of galaxy groups and clusters with cavities: the relationship between jet power and low-frequency radio emission," Astrophysical Journal Letters, vol. 735, article 11, no. 1, 2011.

[166] W. G. Mathews and F. Brighenti, "Creation of the X-ray cavity jet and its radio lobe in M87/Virgo with cosmic rays: relevance to relic radio sources," Astrophysical Journal, vol. 676, no. 2, pp. 880-888, 2008.

[167] W. G. Mathews and F. Brighenti, "Energetics of X-ray cavities and radio lobes in galaxy clusters," Astrophysical Journal, vol. 685, no. 1, pp. 128-137, 2008.

[168] P. J. Mendygral, S. M. O’Neill, and T. W. Jones, "Synthetic observations of simulated active galactic nucleus jets: X-ray cavities," Astrophysical Journal Letters, vol. 730, article 100, no. 2, 2011.

[169] K. W. Cavagnolo, B. R. McNamara, P. E. J. Nulsen, C. L. Carilli, C. Jones, and L. Bîrzan, "A relationship between agn jet power and radio power," Astrophysical Journal, vol. 720, no. 2, pp. 1066-1072, 2010.

[170] C. Nipoti and J. Binney, "Time variability of active galactic nuclei and heating of cooling flows," Monthly Notices of the Royal Astronomical Society, vol. 361, no. 2, pp. 428-436, 2005.

[171] R. J. H. Dunn and A. C. Fabian, "Investigating heating and cooling in the BCS and B55 cluster samples," Monthly Notices of the Royal Astronomical Society, vol. 385, no. 2, pp. 757-768, 2008.

[172] E. C. D. Pope, G. Pavlovski, C. R. Kaiser, and H. Fangohr, "Heating rate profiles in galaxy clusters," Monthly Notices of the Royal Astronomical Society, vol. 367, no. 3, pp. 1121-1131, 2006.

[173] R. J. H. Dunn, W. Allen, G. B. Taylor et al., "The radio properties of a complete, X-ray selected sample of nearby, massive elliptical galaxies," Monthly Notices of the Royal Astronomical Society, vol. 404, no. 1, pp. 180-197, 2010.

[174] F. Brighenti and W. G. Mathews, "Heated cooling flows," Astrophysical Journal, vol. 573, no. 2, pp. 542-561, 2002.

[175] C. J. Willott, S. Rawlings, K. M. Blundell, and M. Lacy, “The emission line-radio correlation for radio sources using the 7C Redshift Survey," Monthly Notices of the Royal Astronomical Society, vol. 309, no. 4, pp. 1017-1033, 1999.

[176] A. G. Pacholczyk, Radio Astrophysics. Nonthermal Processes in Galactic and Extragalactic Sources, 1970.

[177] G. Brunetti, "Non-thermal emission from extragalactic radio sources: a high resolution broad band (radio to X-rays) approach," http://arxiv.org/abs/astro-ph/0207671.

[178] G. Brunetti, G. Setti, and A. Comastri, "Inverse Compton X-rays from strong FRII radio-galaxies," Astronomy and Astrophysics, vol. 325, no. 3, pp. 898-910, 1997.

[179] D. S. De Young, "The particle content of extragalactic jets," Astrophysical Journal, vol. 648, no. 1, pp. 200-208, 2006.

[180] J. H. Croston, M. J. Hardcastle, M. Birkinshaw, D. M. Worrall, and R. A. Laing, "An XMM-Newton study of the environments, particle content and impact of low-power radio galaxies," Monthly Notices of the Royal Astronomical Society, vol. 386, no. 3, pp. 1709-1728, 2008.

[181] R. J. H. Dunn and A. C. Fabian, "Particle energies and filling fractions of radio bubbles in cluster cores," Monthly Notices of the Royal Astronomical Society, vol. 355, no. 3, pp. 862-873, 2004.

[182] R. J. H. Dunn, A. C. Fabian, and A. Celotti, "Using radio bubbles to constrain the matter content of AGN jets," Monthly Notices of the Royal Astronomical Society, vol. 372, no. 4, pp. 1741-1748, 2006.

[183] M. Gitti, G. Brunetti, L. Feretti, and G. Setti, "Particle acceleration in cooling flow clusters of galaxies: the case of Abell 2626," Astronomy and Astrophysics, vol. 417, no. 1, pp. $1-11,2004$.

[184] M. Gitti, C. Ferrari, W. Domainko, L. Feretti, and S. Schindler, "Discovery of diffuse radio emission at the center of the most X-ray-luminous cluster RX J1347.5-1145," Astronomy and Astrophysics, vol. 470, no. 3, pp. L25-L28, 2007.

[185] H. Böhringer, V. Burwitz, Y. Y. Zhang, P. Schuecker, and N. Nowak, "Chandra reveals galaxy cluster with the most massive nearby cooling core: RXC J1504.1-0248," Astrophysical Journal, vol. 633, no. 1, pp. 148-153, 2005.

[186] S. Giacintucci, M. Markevitch, G. Brunetti, R. Cassano, and T. Venturi, "A radio minihalo in the extreme cool-core galaxy cluster RXC J1504.1-0248," Astronomy and Astrophysics, vol. 525, no. 7, article L10, 2011.

[187] M. Gitti, G. Brunetti, and G. Setti, "Modeling the interaction between ICM and relativistic plasma in cooling flows: the case of the Perseus cluster," Astronomy and Astrophysics, vol. 386, no. 2, pp. 456-463, 2002.

[188] J. O. Burns, M. E. Sulkanen, G. R. Gisler, and R. A. Perley, "Where have all the cluster halos gone?" Astrophysical Journal, vol. 388, no. 2, pp. L49-L52, 1992.

[189] E. Rizza, C. Loken, M. Bliton, K. Roettiger, J. O. Burns, and F. N. Owen, "X-ray and radio interactions in the cores of cooling flow clusters," Astronomical Journal, vol. 119, no. 1, pp. 21-31, 2000.

[190] G. Giovannini and L. Feretti, "Halo and relic sources in clusters of galaxies," New Astronomy, vol. 5, no. 6, pp. 335$347,2000$. 
[191] S. A. Baum and C. P. O’Dea, "Multifrequency VLA observations of PKS 0745-191 - the archetypal 'cooling flow' radio source?" Monthly Notices of the Royal Astronomical Society, vol. 250, pp. 737-749, 1991.

[192] M. Bacchi, L. Feretti, G. Giovannini, and F. Govoni, "Deep images of cluster radio halos," Astronomy and Astrophysics, vol. 400, no. 2, pp. 465-476, 2003.

[193] T. Venturi, S. Giacintucci, D. Dallacasa et al., "GMRT radio halo survey in galaxy clusters at $\mathrm{z}=0.2-0.4$ II. the eBCS clusters and analysis of the complete sample," Astronomy and Astrophysics, vol. 484, no. 2, pp. 327-340, 2008.

[194] F. Govoni, M. Murgia, M. Markevitch et al., "A search for diffuse radio emission in the relaxed, cool-core galaxy clusters A1068, A1413, A1650, A1835, A2029, and Ophiuchus," Astronomy and Astrophysics, vol. 499, no. 2, pp. 371-383, 2009.

[195] M. Murgia, D. Eckert, F. Govoni et al., "GMRT observations of the Ophiuchus galaxy cluster," Astronomy and Astrophysics, vol. 514, no. 12, article 76, 2010.

[196] C. Pfrommer and T. A. Enßlin, "Constraining the population of cosmic ray protons in cooling flow clusters with $\gamma$-ray and radio observations: are radio mini-halos of hadronic origin?" Astronomy and Astrophysics, vol. 413, no. 1, pp. 17-36, 2004.

[197] U. Keshet and A. Loeb, "Using radio halos and minihalos to measure the distributions of magnetic fields and cosmic rays in galaxy clusters," Astrophysical Journal, vol. 722, no. 1, pp. 737-749, 2010.

[198] R. Cassano, M. Gitti, and G. Brunetti, "A morphological comparison between giant radio halos and radio mini-halos in galaxy clusters," Astronomy and Astrophysics, vol. 486, no. 3, pp. L31-L34, 2008.

[199] P. Mazzotta and S. Giacintucci, "Do radio core-halos and cold fronts in non-major-merging clusters originate from the same gas sloshing?" Astrophysical Journal, vol. 675, no. 1, pp. L9-L12, 2008.

[200] J. ZuHone, M. Markevitch, and G. Brunetti, "Testing the connection between radio mini-halos and core gas sloshing with MHD simulations," http://arxiv.org/abs/1101.4627.

[201] S. Heinz, "The interaction of relativistic jets with their environment," New Astronomy Reviews, vol. 47, no. 6-7, pp. 565-567, 2003.

[202] F. Brighenti and W. G. Mathews, "Stopping cooling flows with jets," Astrophysical Journal, vol. 643, no. 1, pp. 120-127, 2006.

[203] M. Gaspari, C. Melioli, F. Brighenti, and A. D'Ercole, “The dance of heating and cooling in galaxy clusters: threedimensional simulations of self-regulated active galactic nuclei outflows," Monthly Notices of the Royal Astronomical Society, vol. 411, no. 1, pp. 349-372, 2011.

[204] R. P. Kraft, P. E. J. Nulsen, M. Birkinshaw et al., “A Chandra study of the lobe/interstellar medium interactions around the inner radio lobes of centaurus A: constraints on the temperature structure and transport processes," Astrophysical Journal, vol. 665, no. 2, pp. 1129-1137, 2007.

[205] J. H. Croston, R. P. Kraft, M. J. Hardcastle et al., "High-energy particle acceleration at the radio-lobe shock of Centaurus A," Monthly Notices of the Royal Astronomical Society, vol. 395, no. 4, pp. 1999-2012, 2009.

[206] P. E. J. Nulsen, D. C. Hambrick, B. R. McNamara et al., "The powerful outburst in Hercules A," Astrophysical Journal, vol. 625, no. 1, pp. L9-L12, 2005.
[207] K. K. S. Wu, A. C. Fabian, and P. E. J. Nulsen, "Non-gravitational heating in the hierarchical formation of X-ray clusters," Monthly Notices of the Royal Astronomical Society, vol. 318, no. 3, pp. 889-912, 2000.

[208] E. Puchwein, D. Sijacki, and V. Springel, "Simulations of AGN feedback in galaxy clusters and groups: impact on gas fractions and the L-T scaling relation," Astrophysical Journal, vol. 687, pp. L53-L56, 2008.

[209] M. Gitti, B. R. McNamara, P. E. J. Nulsen, and M. W. Wise, "Cosmological effects of powerful AGN outbursts in galaxy clusters: insights from an XMM-Newton observation of MS 0735+7421," Astrophysical Journal, vol. 660, no. 2, pp. 11181136, 2007.

[210] A. E. Evrard, C. A. Metzler, and J. F. Navarro, "Mass estimates of X-ray clusters," Astrophysical Journal, vol. 469, no. 2, pp. 494-507, 1996.

[211] D. Lynden-Bell, "Galactic nuclei as collapsed old quasars," Nature, vol. 223, no. 5207, pp. 690-694, 1969.

[212] M. C. Begelman, R. D. Blandford, and M. J. Rees, "Theory of extragalactic radio sources," Reviews of Modern Physics, vol. 56, no. 2, pp. 255-351, 1984.

[213] R. Narayan and J. E. McClintock, "Advection-dominated accretion and the black hole event horizon," New Astronomy Reviews, vol. 51, no. 10-12, pp. 733-751, 2008.

[214] A. J. Barger, L. L. Cowie, M. W. Bautz et al., "Supermassive black hole accretion history inferred from a large sample of Chandra hard X-ray sources," Astronomical Journal, vol. 122, no. 5, pp. 2177-2189, 2001.

[215] J. Hlavacek-Larrondo and A. C. Fabian, "Investigating a sample of strong cool core, highly luminous clusters with radiatively inefficient nuclei," Monthly Notices of the Royal Astronomical Society, vol. 413, pp. 313-321, 2011.

[216] B. R. McNamara, F. Kazemzadeh, D. A. Rafferty et al., "An energetic AGN outburst powered by a rapidly spinning supermassive black hole or an accreting ultramassive black hole," Astrophysical Journal, vol. 698, no. 1, pp. 594-605, 2009.

[217] E. C. D. Pope, A. Babul, G. Pavlovski, R. G. Bower, and A. Dotter, "Mass transport by buoyant bubbles in galaxy clusters," Monthly Notices of the Royal Astronomical Society, vol. 406, no. 3, pp. 2023-2037, 2010.

[218] C. S. Reynolds, E. A. Casper, and S. Heinz, "A deep Chandra observation of Abell 4059: a new face to "radio-mode" AGN feedback?" Astrophysical Journal, vol. 679, no. 2, pp. 11811191, 2008.

[219] A. Simionescu, N. Werner, H. Böhringer et al., "Chemical enrichment in the cluster of galaxies Hydra A," Astronomy and Astrophysics, vol. 493, no. 2, pp. 409-424, 2009.

[220] N. Werner, A. Simionescu, E. T. Million et al., "Feedback under the microscope-II. Heating, gas uplift and mixing in the nearest cluster core," Monthly Notices of the Royal Astronomical Society, vol. 407, no. 4, pp. 2063-2074, 2010.

[221] C. C. Kirkpatrick, B. R. McNamara, and K. W. Cavagnolo, "Anisotropic metal-enriched outflows driven by active galactic nuclei in clusters of galaxies," Astrophysical Journal Letters, vol. 731, article L23, no. 2, 2011.

[222] C. S. Reynolds, S. Heinz, and M. C. Begelman, "The hydrodynamics of dead radio galaxies," Monthly Notices of the Royal Astronomical Society, vol. 332, no. 2, pp. 271-282, 2002.

[223] M. Ruszkowski, M. Brüggen, and M. C. Begelman, "Cluster heating by viscous dissipation of sound waves," Astrophysical Journal, vol. 611, no. 1, pp. 158-163, 2004. 
[224] T. W. Jones and D. S. De Young, "Magnetohydrodynamic simulations of relic radio bubbles in clusters," Astrophysical Journal, vol. 624, no. 2, pp. 586-605, 2005.

[225] N. Soker and F. Pizzolato, "Feedback heating with slow jets in cooling flow clusters," Astrophysical Journal, vol. 622, no. 2, pp. 847-852, 2005.

[226] S. Heinz, M. Briiggen, A. Young, and E. Levesque, "The answer is blowing in the wind: simulating the interaction of jets with dynamic cluster atmospheres," Monthly Notices of the Royal Astronomical Society, vol. 373, no. 1, pp. L65-L69, 2006.

[227] M. Brüggen, S. Heinz, E. Roediger, M. Ruszkowski, and A. Simionescu, "Shock heating by Fanaroff-Riley type I radio sources in galaxy clusters," Monthly Notices of the Royal Astronomical Society, vol. 380, no. 1, pp. L67-L70, 2007.

[228] A. Sternberg, F. Pizzolato, and N. Soker, "Inflating fat bubbles in clusters of galaxies by wide jets," Astrophysical Journal, vol. 656, no. 1, pp. L5-L8, 2007.

[229] M. Brüggen and E. Scannapieco, "Self-regulation of active galactic nuclei in galaxy clusters," Monthly Notices of the Royal Astronomical Society, vol. 398, no. 2, pp. 548-560, 2009.

[230] B. J. Morsony, S. Heinz, M. Brüggen, and M. Ruszkowski, "Swimming against the current: simulations of central AGN evolution in dynamic galaxy clusters," Monthly Notices of the Royal Astronomical Society, vol. 407, no. 2, pp. 1277-1289, 2010.

[231] M. Ruszkowski and S. P. Oh, "Galaxy motions, turbulence and conduction in clusters of galaxies," Monthly Notices of the Royal Astronomical Society, vol. 414, no. 2, pp. 1493-1507, 2011.

[232] H. Omma, J. Binney, G. Bryan, and A. Slyz, "Heating cooling flows with jets," Monthly Notices of the Royal Astronomical Society, vol. 348, no. 4, pp. 1105-1119, 2004.

[233] C. Zanni, G. Murante, G. Bodo, S. Massaglia, P. Rossi, and A. Ferrari, "Heating groups and clusters of galaxies: the role of AGN jets," Astronomy and Astrophysics, vol. 429, no. 2, pp. 399-415, 2005.

[234] J. C. Vernaleo and C. S. Reynolds, "AGN feedback and cooling flows: problems with simple hydrodynamic models," Astrophysical Journal, vol. 645, no. 1, pp. 83-94, 2006.

[235] A. Cattaneo and R. Teyssier, "AGN self-regulation in cooling flow clusters," Monthly Notices of the Royal Astronomical Society, vol. 376, no. 4, pp. 1547-1556, 2007.

[236] M. Brüggen, "The effect of mixing on metallicity gradients in the intracluster medium," Astrophysical Journal, vol. 571, no. 1, pp. L13-L16, 2002.

[237] E. Roediger, M. Brüggen, P. Rebusco, H. Böhringer, and E. Churazov, "Metal mixing by buoyant bubbles in galaxy clusters," Monthly Notices of the Royal Astronomical Society, vol. 375, no. 1, pp. 15-28, 2007. 

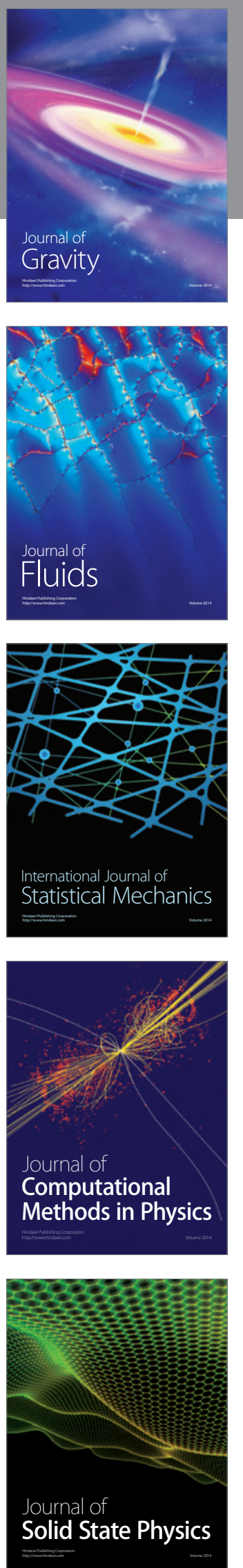

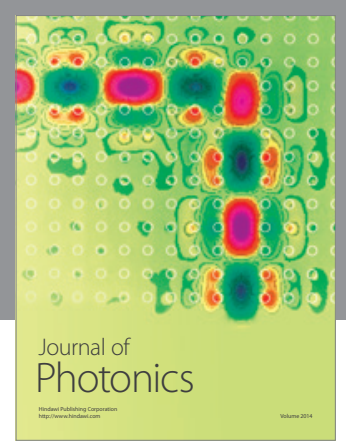

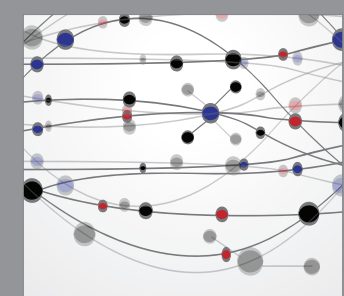

The Scientific World Journal
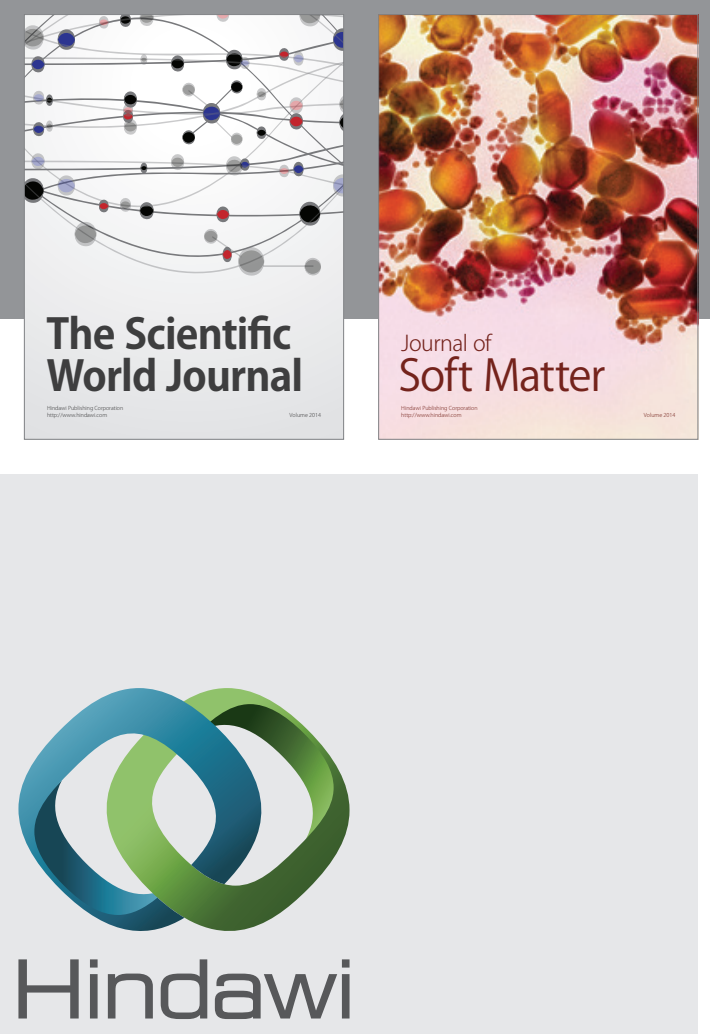

Submit your manuscripts at

http://www.hindawi.com
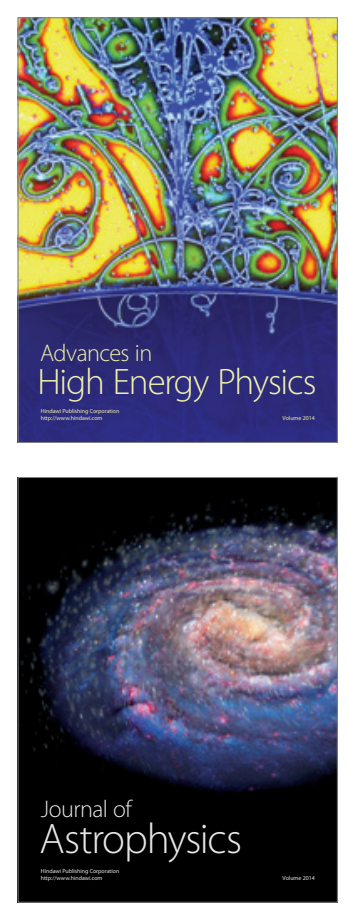
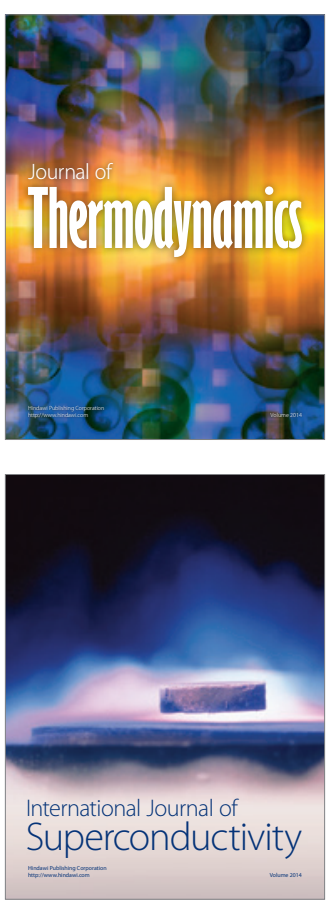
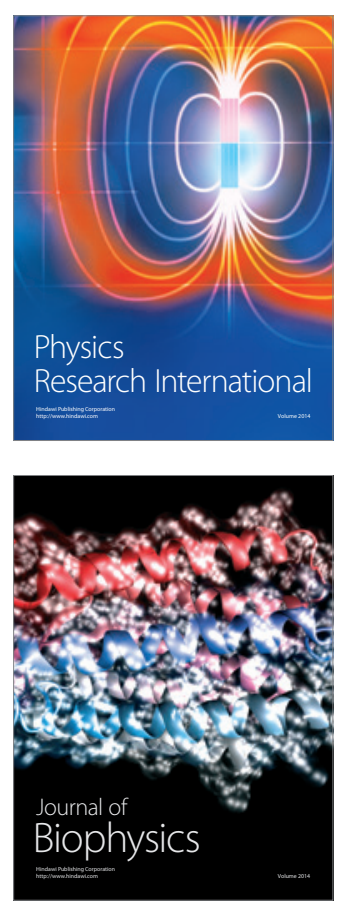
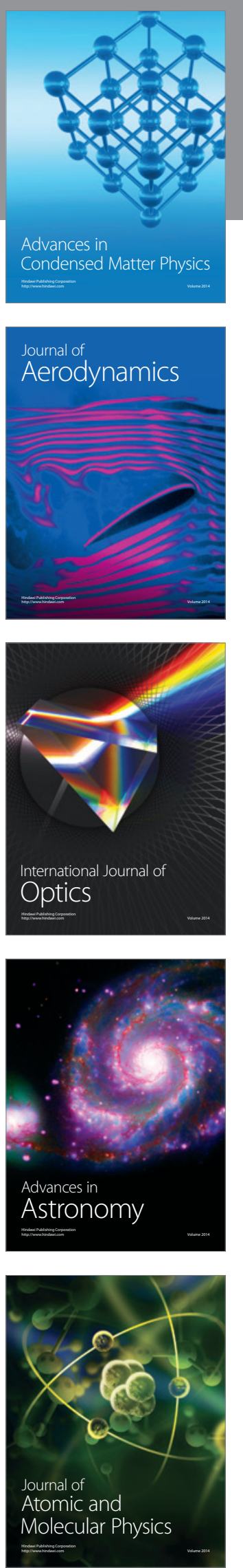